\title{
Modeling interval time series with Space-time processes
}

\author{
Paulo Teles and Paula Brito
}

Faculdade de Economia \& LIAAD-INESC Porto LA, University of Porto

Rua Dr. Roberto Frias, 4200-464 Porto, Portugal

\section{Corresponding author:}

Paulo Teles

Faculdade de Economia \& LIAAD-INESC Porto LA, University of Porto

Rua Dr. Roberto Frias, 4200-464 Porto, Portugal

Email: pteles@fep.up.pt

Short Title: Space-time processes for interval time series 
Abstract. We consider interval-valued time series, i.e., series resulting from collecting real intervals as an ordered sequence through time. Since the lower and upper bounds of the observed intervals at each time point are in fact values of the same variable, they are naturally related. We propose modeling interval-time series with Space-time autoregressive models and, based on the process appropriate for the interval bounds, we derive the model for the intervals' center and radius. A simulation study and an application with data of daily wind speed at different meteorological stations in Ireland illustrate that the proposed approach is appropriate and useful.

Keywords. Interval time series; Interval data; Space-time AR model; Prediction.

\section{Introduction}

In classical data analysis, variables describing entities are usually mono-valued, that is, each entity takes exactly one value for each variable at each point in time. However, this model is too restrictive to represent complex data which may comprehend variability and/or uncertainty. The need to consider data containing information that cannot be represented within the classical framework led to the development of Symbolic Data Analysis. Symbolic data extend the classical model, where each individual takes exactly one value for each variable, by allowing multiple, possibly weighted, values for each variable. New variable types have been introduced enabling us to represent variability and/or uncertainty inherent to the data: multi-valued variables, interval variables and modal variables (Bock and Diday, 2000). A variable is called set-valued if its values are nonempty subsets of the underlying domain; it is multi-valued if its values are finite subsets of the domain and it is an interval variable if its values are intervals of $\mathbb{R}$. A survey of this new area can be found in Billard and Diday (2006), Diday and Noirhomme (2008), or, more recently, in Noirhomme and Brito (2011).

Interval data refers to data sets where the observed values of the variables are intervals of $\mathbb{R}$ and may arise in multiple situations that result from temporal aggregation or systematic sampling such as recording monthly temperatures or the daily wind speed in different locations or daily stock prices or returns. Other important sources of interval data are the aggregation of huge data-bases in groups or classes, when the individual real values are generalized by intervals, or situations where there is some inaccuracy or uncertainty 
in recording the value of a (classical) variable (e.g., due to measurement errors). Intervalvalued data may be represented by the lower $\left(l_{i}\right)$ and upper $\left(u_{i}\right)$ bounds of each observed interval $I_{i}=\left[l_{i}, u_{i}\right], i=1, \ldots, n$, or, alternatively, by its center $c_{i}=\frac{l_{i}+u_{i}}{2}$ and radius $r_{i}=\frac{u_{i}-l_{i}}{2}$.

Many different approaches have now been developed to analyze interval data. Assuming a uniform distribution in each observed interval, Bertrand and Goupil (2000) have proposed central tendency and dispersion measures for interval data. Billard and Diday (2003) developed this approach introducing association measures. Duarte Silva and Brito (2006) discuss the properties of dispersion and association measures for interval data and their implications in the definition of linear combinations of interval-valued variables. Based on the uniform assumption, Billard and Diday (2000) have proposed a regression model for interval-valued data. Principal Component Analysis of interval data has first been addressed by Chouakria et al (2000) either by representing the observed intervals by their centers ("centers method") or by considering all the vertices of the hypercube representing each of the $n$ individuals in a $p$-dimensional space ("vertices method"). A different approach is based on representing each variable by the midpoints and ranges of its interval values, a line of work followed by Lauro and Palumbo (2005) on Principal Component Analysis and also by Neto et al (2008, 2010) on Regression Analysis. In Duarte Silva and Brito (2006) different approaches for Linear Discriminant Analysis of interval data are investigated.

When the interval-valued data such as in the examples mentioned above are collected as an ordered sequence through time (daily, monthly, quarterly, etc) or any other dimension, they form a time series and consequently the appropriate methodology to study and model them is time series analysis. To describe time series data, ARIMA (Autoregressive Integrated Moving Average) models are usually considered (Box, Jenkins and Reinsel, 2008; Brockwell and Davis, 2002; Wei, 2006) but these are appropriate for single-valued data sets. New developments to model interval time series have recently been proposed.

Teles and Brito (2005) were the first to consider interval-valued time series data using an approach based on fitting univariate ARIMA processes to the interval bounds (see also Brito, 2007). Maia, De Carvalho and Ludermir (2008) propose fitting univariate ARIMA processes applied to the center and radius and use them to forecast the interval bounds, 
since the latter can be obtained from the former. They also propose an approach based on an artificial neural network model as well as a combination of both.

Arroyo (2008), García-Ascanio and Maté (2009), González-Rivera and Arroyo (2011) and Han et al. (2008) define interval stochastic process, interval-valued time series, weak stationarity for interval processes and, based on the sample moments previously proposed (Bertrand and Goupil, 2000), also define the empirical autocovariance and autocorrelation functions for interval time series data, aiming at uncovering the data-generating process behind this type of symbolic data sets. Arroyo (2008), García-Ascanio and Maté (2009) and Arroyo, González-Rivera and Maté (2010) focus on forecasting based on Vector Autoregressive models (VAR), Vector Error Correction models (VEC) and smoothing filters.

Other approaches may provide a new insight into modeling interval time series data, particularly concerning its description, understanding and forecasting. In this paper, we propose modeling such data with Space-time processes, thus taking into account the existence of contemporaneous correlation or dependence between the intervals' lower and upper bounds (or center and radius). The main contribution of the paper is therefore the proposal of Space-time models as an adequate approach to model interval time series.

In the next section we introduce interval time series and review the main models used in the literature. Section 3 presents the class of Space-time autoregressive models which are then used in Section 4 to model interval time series. In Section 5, a simulation experiment is conducted to compare the predictive performance of the different approaches considered and Section 6 describes an application to real data. Finally, concluding remarks are given in Section 7.

\section{Interval time series}

An interval time series (ITS) is a realization of an interval stochastic process (see the references mentioned above), i.e., for each moment in time $t=1, \ldots, N$, let $X_{L_{t}}$ and $X_{U_{t}}$ denote the lower and the upper bounds of the observed interval, with $X_{L_{t}} \leq X_{U_{t}}$; the interval time series is then

$$
\left[X_{L_{1}}, X_{U_{1}}\right],\left[X_{L_{2}}, X_{U_{2}}\right], \ldots,\left[X_{L_{N}}, X_{U_{N}}\right]
$$


We assume that $X_{L_{t}}$ and $X_{U_{t}}$ are both weakly stationary, which implies the weak stationarity of the interval time series (see the references mentioned above). Let also $C_{t}$ and $R_{t}$ denote the center and the radius of the ITS at time $t$ respectively, i.e., $C_{t}=\frac{X_{L_{t}}+X_{U_{t}}}{2}$ and $R_{t}=\frac{X_{U_{t}}-X_{L_{t}}}{2}$. Weak stationarity of $X_{L_{t}}$ and $X_{U_{t}}$ implies that of $C_{t}$ and $R_{t}$.

Arroyo (2008) and García-Ascanio and Maté (2009) propose the following Vector Autoregressive model of order $p(\operatorname{VAR}(p))$ for ITS:

$$
\begin{aligned}
& X_{L_{t}}=\beta_{L}+\sum_{i=1}^{p} \beta_{L, i}^{L} X_{L_{t-i}}+\sum_{i=1}^{p} \beta_{L, i}^{U} X_{U_{t-i}}+\xi_{L_{t}} \\
& X_{U_{t}}=\beta_{U}+\sum_{i=1}^{p} \beta_{U, i}^{L} X_{L_{t-i}}+\sum_{i=1}^{p} \beta_{U, i}^{U} X_{U_{t-i}}+\xi_{U_{t}}
\end{aligned}
$$

where $\beta_{L}, \beta_{U}$ are the intercepts, $\beta_{L, i}^{L}, \beta_{L, i}^{U}, \beta_{U, i}^{L}$ and $\beta_{U, i}^{U}(i=1, \ldots, p)$ are the autoregressive parameters and $\xi_{L_{t}}$ and $\xi_{U_{t}}$ are white noise processes with zero mean, variances $\sigma_{\xi_{L}}^{2}$ and $\sigma_{\xi_{U}}^{2}$ respectively, and covariance

$$
\operatorname{Cov}\left(\xi_{L_{t}}, \xi_{U_{t+k}}\right)=\left\{\begin{array}{cl}
\sigma_{\xi_{L U}} & \text { if } k=0 \\
0 & \text { if } k \neq 0
\end{array}\right.
$$

In vector notation, if $\mathbf{X}_{t}=\left(X_{L_{t}}, X_{U_{t}}\right)^{T}, \mathbf{B}=\left(\beta_{L}, \beta_{U}\right)^{T}, \mathbf{B}_{i}=\left(\begin{array}{cc}\beta_{L, i}^{L} & \beta_{L, i}^{U} \\ \beta_{U, i}^{L} & \beta_{U, i}^{U}\end{array}\right)$ and $\boldsymbol{\Xi}_{t}=$ $\left(\xi_{L_{t}}, \xi_{U_{t}}\right)^{T}$ (where $T$ means transpose), model (2) may be written as

$$
\mathbf{X}_{t}=\mathbf{B}+\sum_{i=1}^{p} \mathbf{B}_{i} \mathbf{X}_{t-i}+\mathbf{\Xi}_{t}
$$

When $X_{L_{t}}$ and $X_{U_{t}}$ are cointegrated, a Vector Error Correction Model can be derived.

From model (2), Arroyo (2008) derives the corresponding model of $\left(C_{t}, R_{t}\right)$ and shows it is also a $\operatorname{VAR}(p)$ :

$$
\begin{aligned}
& C_{t}=\beta_{C}+\sum_{i=1}^{p} \beta_{C, i}^{C} C_{t-i}+\sum_{i=1}^{p} \beta_{C, i}^{R} R_{t-i}+\xi_{C_{t}} \\
& R_{t}=\beta_{R}+\sum_{i=1}^{p} \beta_{R, i}^{C} C_{t-i}+\sum_{i=1}^{p} \beta_{R, i}^{R} R_{t-i}+\xi_{R_{t}}
\end{aligned}
$$

which, in vector notation, with $\mathbf{Y}_{t}=\left(C_{t}, R_{t}\right)^{T}$, is written as

$$
\mathbf{Y}_{t}=\boldsymbol{\Lambda}+\sum_{i=1}^{p} \boldsymbol{\Lambda}_{j} \mathbf{Y}_{t-i}+\boldsymbol{\Omega}_{t}
$$


where a similar notation to that of (2) and (3) is adopted. The parameters in models (4) and (5) can be written as functions of those in (2) and (3) respectively. Arroyo (2008) also shows that the models corresponding to (4) or (5) in terms of $\left(X_{L_{t}}, X_{U_{t}}\right)$ or $\mathbf{X}_{t}$ are (2) and (3) respectively. The parameters in the latter models can be written as functions of those in the former. In short, models (2) and (4), or (3) and (5) are equivalent and it is always possible to derive one of them from the other, including their parameters. The parameters in both models can be estimated by ordinary least squares.

Given the data $\mathbf{X}_{1}, \ldots, \mathbf{X}_{N}$ or $\mathbf{Y}_{1}, \ldots, \mathbf{Y}_{N}$, the minimum mean square error predictors at time $t=N$ of the $m$-step ahead $(m=1,2 \ldots)$ future values $\mathbf{X}_{N+m}$ or $\mathbf{Y}_{N+m}$ based on models (2) or (3) and on models (4) or (5) respectively, $\widehat{\mathbf{X}}_{N}(m)$ and $\widehat{\mathbf{Y}}_{N}(m)$, are the conditional expectations (Arroyo, 2008)

$$
\begin{aligned}
\widehat{\mathbf{X}}_{N}(m) & =E\left(\mathbf{X}_{N+m} \mid \mathbf{X}_{N}, \mathbf{X}_{N-1}, \ldots\right) \\
& \Leftrightarrow\left\{\begin{array}{l}
\widehat{X}_{L_{N}}(m)=E\left(X_{L_{N+m}} \mid\left(X_{L_{N}}, X_{U_{N}}\right)^{T},\left(X_{L_{N-1}}, X_{U_{N-1}}\right)^{T}, \ldots\right) \\
\widehat{X}_{U_{N}}(m)=E\left(X_{U_{N+m}} \mid\left(X_{L_{N}}, X_{U_{N}}\right)^{T},\left(X_{L_{N-1}}, X_{U_{N-1}}\right)^{T}, \ldots\right)
\end{array}\right.
\end{aligned}
$$

and

$$
\begin{aligned}
\widehat{\mathbf{Y}}_{N}(m) & =E\left(\mathbf{Y}_{N+m} \mid \mathbf{Y}_{N}, \mathbf{Y}_{N-1}, \ldots\right) \\
& \Leftrightarrow\left\{\begin{array}{l}
\widehat{C}_{N}(m)=E\left(C_{N+m} \mid\left(C_{N}, R_{N}\right)^{T},\left(C_{N-1}, R_{N-1}\right)^{T}, \ldots\right) \\
\widehat{R}_{N}(m)=E\left(R_{N+m} \mid\left(C_{N}, R_{N}\right)^{T},\left(C_{N-1}, R_{N-1}\right)^{T}, \ldots\right) .
\end{array}\right.
\end{aligned}
$$

Thus,

$$
\begin{aligned}
\widehat{X}_{L_{N}}(m) & =\widehat{C}_{N}(m)-\widehat{R}_{N}(m) \\
\widehat{X}_{U_{N}}(m) & =\widehat{C}_{N}(m)+\widehat{R}_{N}(m) \\
\widehat{C}_{N}(m) & =\frac{\widehat{X}_{L_{N}}(m)+\widehat{X}_{U_{N}}(m)}{2} \\
\widehat{R}_{N}(m) & =\frac{\widehat{X}_{U_{N}}(m)-\widehat{X}_{L_{N}}(m)}{2} .
\end{aligned}
$$

All these approaches treat the interval bounds as two different univariate time series and propose a model to describe their relationship. However, this may not be the most 
appropriate since interval data result from observations of the same variable made at different points. Therefore, an approach that takes this important feature of interval time series data into account is required.

\section{Space-time AR models}

The basic format of the data in the examples mentioned in the introduction is the recording of observations of a variable as an ordered sequence through time at different spatial locations for each period of time. Thus, the class of Space-time processes is a natural framework to model those data sets and in fact it is able to describe these as sets of observations of the same variable made at different points. Some definitions are required first.

Let $\mathbf{s}=\left(s_{1}, \ldots, s_{m}\right) \in D \subset \mathrm{IR}^{m}$, where $D$ is an index set, be a generic data (spatial) location in a $m$-dimensional Euclidean space and let $t \in T$ where $T$, an index set, is the set of time points. A space-time process is the multivariate random field $\left\{X_{\mathbf{s}, t}: \mathbf{s} \in D, t \in T\right\}$, where it is also possible to have $D(t)$ (Cressie, 1993). Usually, $m=2$, i.e., $\mathbf{s}=\left(s_{1}, s_{2}\right) \in$ $D \subset \mathrm{IR}^{2}$ is the spatial location and $s_{1}$ and $s_{2}$ are the space coordinates, but $m=3$, i.e., $\mathbf{s}=\left(s_{1}, s_{2}, s_{3}\right) \in D \subset \mathrm{IR}^{3}$ or $m=1$, i.e., $s \in D \subset \mathrm{IR}$ also tipically occur. We will assume that $D$ is a fixed finite set and that the index set $T$ is the set of all integers, $T=\{0, \pm 1, \pm 2, \ldots\}$. Suppose then that $X_{\mathbf{s}_{i}, t}$ is an observation taken at time $t$, with $t=1, \ldots, N$, and spatial location $\mathbf{s}_{i}$, with $i=1, \ldots, n$, i.e., there are $n$ locations, the number of elements of $D$. Let $\left\{X_{\mathbf{s}_{i}, t}: i=1, \ldots, n ; t=1, \ldots, N\right\}$ denote the data set and $\mathbf{X}_{t}=\left(X_{\mathbf{s}_{1}, t}, \ldots, X_{\mathbf{s}_{n}, t}\right)^{T}$.

Space-time autoregressive (STAR) models (Cliff and Ord, 1975; Pfeifer and Deutsch, 1980; Cressie, 1993) generalize the time-series AR models by incorporating the spatial dimension and are therefore characterized by linear dependence in both space and time (see also Antunes and Subba Rao, 2006; Diggle and Ribeiro, 2007; Finkenstädt, Held and Isham, 2007; Le and Zidek, 2006). A stationary $\operatorname{STAR}(p)$ process is written as

$$
\mathbf{X}_{t}=\boldsymbol{\Theta}+\psi_{0} \mathbf{W} \mathbf{X}_{t}+\sum_{i=1}^{p}\left(\phi_{i} \mathbf{I}_{n}+\psi_{i} \mathbf{W}\right) \mathbf{X}_{t-i}+\mathbf{a}_{t}
$$

where $\boldsymbol{\Theta}$ is a $(n \times 1)$ vector of intercepts, the scalars $\psi_{i}(i=0, \ldots, p)$ and $\phi_{j}(j=1, \ldots, p)$ are unknown parameters, $\mathbf{I}_{n}$ is the $(n \times n)$ identity matrix and $\mathbf{W}$ is a non-zero $(n \times n)$ 
matrix of known weights with diagonal elements 0 and off-diagonal elements (the weights) usually non-negative and related to the distances between the spatial locations. This matrix, with elements $\omega_{i j}(i, j=1, \ldots, n)$, may be either symmetric $\left(\omega_{i j}=\omega_{j i}\right)$ or not. The weights may be standardized so that each row of $\mathbf{W}$ sums up to 1 . The independent white noise random vector $\mathbf{a}_{t}=\left(a_{\mathbf{s}_{1}, t}, \ldots, a_{\mathbf{s}_{n}, t}\right)^{T}$ is a vector of independent white noise processes at space-time locations $\left\{\left(\mathbf{s}_{i}, t\right): i=1, \ldots, n ; t=1, \ldots, N\right\}$ and has zero mean and covariance matrix

$$
E\left(\mathbf{a}_{t} \mathbf{a}_{t+k}^{T}\right)=\left\{\begin{array}{ccc}
\boldsymbol{\Sigma}_{a} & \text { if } & k=0 \\
\mathbf{0} & \text { if } & k \neq 0
\end{array}\right.
$$

where $\boldsymbol{\Sigma}_{a}$ is a $(n \times n)$ symmetric positive definite matrix. The parameters can be estimated by appropriate methods for simultaneous-equation models, such as two or three-stage least squares, generalized method of moments and, assuming gaussianity, maximum likelihood (Johnston, 1997). A STAR process can easily be generalized to accommodate temporal nonstationarity which can be removed by differencing $\mathbf{X}_{t}$. In such cases, denoting the differencing order by $d,(1-B)^{d} \mathbf{X}_{t}$ follows the $\operatorname{STAR}(p)$ model (8) above.

The STAR model (8) can be used for prediction. Given the data $\mathbf{X}_{1}, \ldots, \mathbf{X}_{N}$, the conditional expectation $\widehat{\mathbf{X}}_{N}(m)=E\left(\mathbf{X}_{N+m} \mid \mathbf{X}_{N}, \mathbf{X}_{N-1}, \ldots\right)$ is the minimum mean square error predictor of $\mathbf{X}_{N+m}$. Therefore, from (8), and letting $E\left(\cdot \mid \mathbf{X}_{N}, \mathbf{X}_{N-1}, \ldots\right)=E_{N}(\cdot)$, the predictor is $(m=1,2, \ldots)$

$$
\widehat{\mathbf{X}}_{N}(m)=\boldsymbol{\Theta}+\psi_{0} \mathbf{W} E_{N}\left(\mathbf{X}_{N+m}\right)+\sum_{i=1}^{p}\left(\phi_{i} \mathbf{I}_{n}+\psi_{i} \mathbf{W}\right) E_{N}\left(\mathbf{X}_{N+m-i}\right)+E_{N}\left(\mathbf{a}_{N+m}\right)
$$

where, for $i=0, \ldots, p$,

$$
E_{N}\left(\mathbf{X}_{N+m-i}\right)=\left\{\begin{array}{ll}
\mathbf{X}_{N+m-i} & m-i \leq 0 \\
\widehat{\mathbf{X}}_{N}(m-i) & m-i>0
\end{array} \text { and } E_{N}\left(\mathbf{a}_{N+m}\right)=\mathbf{0}\right.
$$

Therefore, since $E_{N}\left(\mathbf{X}_{N+m}\right)=\widehat{\mathbf{X}}_{N}(m)$, the predictor (9) above becomes

$$
\widehat{\mathbf{X}}_{N}(m)=\left(\mathbf{I}_{n}-\psi_{0} \mathbf{W}\right)^{-1}\left(\boldsymbol{\Theta}+\sum_{i=1}^{p}\left(\phi_{i} \mathbf{I}_{n}+\psi_{i} \mathbf{W}\right) E_{N}\left(\mathbf{X}_{N+m-i}\right)\right)
$$

assuming $\left(\mathbf{I}_{n}-\psi_{0} \mathbf{W}\right)$ is nonsingular. 


\section{STAR models applied to interval time series}

As mentioned above, the STAR model (8) appropriately takes into account the fact that the interval time series (1) is an ordered sequence of observations of the same variable recorded through time at different data points for each time period, for each of which the lower and upper bounds are retained (and is therefore a suitable data-generating process for an ITS).

Then, let the interval stochastic process $\mathbf{X}_{t}=\left(X_{\mathbf{s}_{L}, t}, X_{\mathbf{S}_{U}, t}\right)^{T}$, where $X_{\mathbf{s}_{L}, t}$ and $X_{\mathbf{s}_{U}, t}$ denote the lower and upper bounds respectively, be the $\operatorname{STAR}(p)$ model with $n=2$ :

$$
\begin{aligned}
& X_{\mathbf{s}_{L}, t}=\theta_{L}+\sum_{i=1}^{p} \phi_{i} X_{\mathbf{s}_{L}, t-i}+\sum_{i=0}^{p} \psi_{i} \omega_{U} X_{\mathbf{s}_{U}, t-i}+a_{\mathbf{s}_{L}, t} \\
& X_{\mathbf{s}_{U}, t}=\theta_{U}+\sum_{i=1}^{p} \phi_{i} X_{\mathbf{s}_{U}, t-i}+\sum_{i=0}^{p} \psi_{i} \omega_{L} X_{\mathbf{s}_{L}, t-i}+a_{\mathbf{s}_{U}, t}
\end{aligned}
$$

where $\theta_{L}, \theta_{U}$ are the intercepts, $\phi_{i}(i=1, \ldots, p)$ and $\psi_{i}(i=0, \ldots, p)$ are the autoregressive parameters, $\omega_{U}$ and $\omega_{L}$ are the weights such that $\mathbf{W}=\left(\begin{array}{cc}0 & \omega_{U} \\ \omega_{L} & 0\end{array}\right)$ and $a_{\mathbf{s}_{L}, t}$ and $a_{\mathbf{s}_{U}, t}$ are white noise processes with zero mean and variances $\sigma_{a_{L}}^{2}$ and $\sigma_{a_{U}}^{2}$ respectively, and covariance

$$
\operatorname{Cov}\left(a_{\mathbf{s}_{L}, t}, a_{\mathbf{s}_{U}, t+k}\right)=\left\{\begin{array}{cl}
\sigma_{a_{L U}} & \text { if } k=0 \\
0 & \text { if } k \neq 0
\end{array}\right.
$$

We note the contemporaneous dependence of each interval bound on the other, an important characteristic to be taken into account in the data and that does not exist in the VAR model (2) nor in the univariate ARIMA models of the center and radius. The interval time series (1) is hence considered a realization of the stochastic process (11) which is given in vector notation by (8) with $n=2, \boldsymbol{\Theta}=\left(\theta_{L}, \theta_{U}\right)^{T}, \mathbf{W}$ as given above and $\mathbf{a}_{t}=\left(a_{\mathbf{s}_{L}, t}, a_{\mathbf{s}_{U}, t}\right)^{T}$.

From (11), we derive in the following proposition the bivariate model of the interval center and radius $C_{t}=\frac{X_{\mathbf{s}_{L}, t}+X_{\mathbf{S}_{U}, t}}{2}$ and $R_{t}=\frac{X_{\mathbf{S}_{U}, t}-X_{\mathbf{s}_{L}, t}}{2}$ respectively, as Arroyo (2008) does for the $\operatorname{VAR}(p)$ model (2) proposed by himself and García-Ascanio and Maté (2009).

Proposition Let $\mathbf{X}_{t}=\left(X_{\mathbf{s}_{L}, t}, X_{\mathbf{S}_{U}, t}\right)^{T}$ be an interval stochastic process, where $X_{\mathbf{S}_{L}, t}$ and $X_{\mathbf{s}_{U}, t}$ denote the lower and upper bounds respectively, following the STAR(p) model (11). Then, denoting by $C_{t}=\left(X_{\mathbf{s}_{L}, t}+X_{\mathbf{s}_{U}, t}\right) / 2$ and $R_{t}=\left(X_{\mathbf{s}_{U}, t}-X_{\mathbf{s}_{L}, t}\right) / 2$ the interval center and radius, respectively, the stochastic process $\mathbf{Y}_{t}=\left(C_{t}, R_{t}\right)^{T}$ follows a bivariate Structural $\operatorname{VAR}$ model, $\operatorname{SVAR}(p)$, whose parameters are functions of those in (11). 
Proof. From model (11), the sum of the interval bounds is

$$
\begin{aligned}
X_{\mathbf{s}_{L}, t}+X_{\mathbf{s}_{U}, t} & =\theta_{L}+\sum_{i=1}^{p} \phi_{i} X_{\mathbf{s}_{L}, t-i}+\sum_{i=0}^{p} \psi_{i} \omega_{U} X_{\mathbf{S}_{U}, t-i}+a_{\mathbf{s}_{L}, t} \\
& +\theta_{U}+\sum_{i=1}^{p} \phi_{i} X_{\mathbf{s}_{U}, t-i}+\sum_{i=0}^{p} \psi_{i} \omega_{L} X_{\mathbf{s}_{L}, t-i}+a_{\mathbf{s}_{U}, t} \\
& =\theta_{L}+\theta_{U}+\sum_{i=1}^{p} \phi_{i}\left(X_{\mathbf{s}_{L}, t-i}+X_{\mathbf{s}_{U}, t-i}\right)+\sum_{i=0}^{p} \psi_{i}\left(\omega_{L} X_{\mathbf{s}_{L}, t-i}+\omega_{U} X_{\mathbf{s}_{U}, t-i}\right) \\
& +a_{\mathbf{s}_{L}, t}+a_{\mathbf{s}_{U}, t}
\end{aligned}
$$

or

$$
\begin{aligned}
\frac{X_{\mathbf{s}_{L}, t}+X_{\mathbf{s}_{U}, t}}{2} & =\frac{\theta_{L}+\theta_{U}}{2}+\sum_{i=1}^{p} \phi_{i} \frac{X_{\mathbf{s}_{L}, t-i}+X_{\mathbf{s}_{U}, t-i}}{2}+\sum_{i=0}^{p} \psi_{i} \frac{\omega_{L} X_{\mathbf{s}_{L}, t-i}+\omega_{U} X_{\mathbf{s}_{U}, t-i}}{2} \\
& +\frac{a_{\mathbf{s}_{L}, t}+a_{\mathbf{S}_{U}, t}}{2}
\end{aligned}
$$

Since $C_{t}=\left(X_{\mathbf{s}_{L}, t}+X_{\mathbf{s}_{U}, t}\right) / 2, R_{t}=\left(X_{\mathbf{s}_{U}, t}-X_{\mathbf{s}_{L}, t}\right) / 2, X_{\mathbf{s}_{U}, t}=C_{t}+R_{t}$ and $X_{\mathbf{s}_{L}, t}=C_{t}-R_{t}$, the above expression is

$$
\begin{aligned}
& C_{t}=\frac{\theta_{L}+\theta_{U}}{2}+\sum_{i=1}^{p} \phi_{i} C_{t-i}+\sum_{i=0}^{p} \frac{\psi_{i}}{2}\left(\omega_{L}\left(C_{t-i}-R_{t-i}\right)+\omega_{U}\left(C_{t-i}+R_{t-i}\right)\right) \\
& +\frac{a_{\mathbf{s}_{L}, t}+a_{\mathbf{s}_{U}, t}}{2} \\
& =\frac{\theta_{L}+\theta_{U}}{2}+\sum_{i=1}^{p} \phi_{i} C_{t-i}+\sum_{i=0}^{p} \psi_{i}\left(\frac{\omega_{L}+\omega_{U}}{2} C_{t-i}+\frac{\omega_{U}-\omega_{L}}{2} R_{t-i}\right) \\
& +\frac{a_{\mathbf{s}_{L}, t}+a_{\mathbf{s}_{U}, t}}{2} \\
& =\frac{\theta_{L}+\theta_{U}}{2}+\psi_{0} \frac{\omega_{L}+\omega_{U}}{2} C_{t}+\sum_{i=1}^{p}\left(\phi_{i}+\psi_{i} \frac{\omega_{L}+\omega_{U}}{2}\right) C_{t-i} \\
& +\sum_{i=0}^{p} \psi_{i} \frac{\omega_{U}-\omega_{L}}{2} R_{t-i}+\frac{a_{\mathbf{s}_{L}, t}+a_{\mathbf{s}_{U}, t}}{2} \\
& \Leftrightarrow C_{t}=\frac{\theta_{L}+\theta_{U}}{2-\psi_{0}\left(\omega_{L}+\omega_{U}\right)}+\sum_{i=1}^{p} \frac{\phi_{i}+\psi_{i} \frac{\omega_{L}+\omega_{U}}{2}}{1-\psi_{0} \frac{\omega_{L}+\omega_{U}}{2}} C_{t-i} \\
& +\sum_{i=0}^{p} \frac{\psi_{i} \frac{\omega_{U}-\omega_{L}}{2}}{1-\psi_{0} \frac{\omega_{L}+\omega_{U}}{2}} R_{t-i}+\frac{a_{\mathbf{s}_{L}, t}+a_{\mathbf{s}_{U}, t}}{2-\psi_{0}\left(\omega_{L}+\omega_{U}\right)} \\
& =\pi_{C}+\sum_{i=1}^{p} \pi_{C, i}^{C} C_{t-i}+\sum_{i=0}^{p} \pi_{C, i}^{R} R_{t-i}+\eta_{C_{t}}
\end{aligned}
$$


where, assuming $\psi_{0} \neq 2 /\left(\omega_{L}+\omega_{U}\right)$,

$$
\begin{aligned}
\pi_{C} & =\frac{\theta_{L}+\theta_{U}}{2-\psi_{0}\left(\omega_{L}+\omega_{U}\right)} ; \quad \pi_{C, i}^{C}=\frac{\phi_{i}+\psi_{i} \frac{\omega_{L}+\omega_{U}}{2}}{1-\psi_{0} \frac{\omega_{L}+\omega_{U}}{2}} i=1, \ldots, p ; \\
\pi_{C, i}^{R} & =\frac{\psi_{i} \frac{\omega_{U}-\omega_{L}}{2}}{1-\psi_{0} \frac{\omega_{L}+\omega_{U}}{2}} \quad i=0, \ldots, p ; \quad \eta_{C_{t}}=\frac{a_{\mathbf{s}_{L}, t}+a_{\mathbf{s}_{U}, t}}{2-\psi_{0}\left(\omega_{L}+\omega_{U}\right)}
\end{aligned}
$$

and $\eta_{C_{t}}$ is an independent white noise process with mean and variance

$$
\begin{aligned}
& E\left(\eta_{C_{t}}\right)=E\left(\frac{a_{\mathbf{s}_{L}, t}+a_{\mathbf{s}_{U}, t}}{2-\psi_{0}\left(\omega_{L}+\omega_{U}\right)}\right)=0 \\
& V\left(\eta_{C_{t}}\right)=\sigma_{\eta_{C}}^{2}=V\left(\frac{a_{\mathbf{s}_{L}, t}+a_{\mathbf{s}_{U}, t}}{2-\psi_{0}\left(\omega_{L}+\omega_{U}\right)}\right)=\frac{\sigma_{a_{L}}^{2}+\sigma_{a_{U}}^{2}+2 \sigma_{a_{L U}}}{\left(2-\psi_{0}\left(\omega_{L}+\omega_{U}\right)\right)^{2}} .
\end{aligned}
$$

Similarly, from (11), the interval range is

$$
\begin{aligned}
X_{\mathbf{s}_{U}, t}-X_{\mathbf{S}_{L}, t} & =\theta_{U}+\sum_{i=1}^{p} \phi_{i} X_{\mathbf{s}_{U}, t-i}+\sum_{i=0}^{p} \psi_{i} \omega_{L} X_{\mathbf{s}_{L}, t-i}+a_{\mathbf{s}_{U}, t} \\
& -\left(\theta_{L}+\sum_{i=1}^{p} \phi_{i} X_{\mathbf{s}_{L}, t-i}+\sum_{i=0}^{p} \psi_{i} \omega_{U} X_{\mathbf{s}_{U}, t-i}+a_{\mathbf{s}_{L}, t}\right) \\
& =\theta_{U}-\theta_{L}+\sum_{i=1}^{p} \phi_{i}\left(X_{\mathbf{s}_{U}, t-i}-X_{\mathbf{s}_{L}, t-i}\right)+\sum_{i=0}^{p} \psi_{i}\left(\omega_{L} X_{\mathbf{s}_{L}, t-i}-\omega_{U} X_{\mathbf{s}_{U}, t-i}\right) \\
& +a_{\mathbf{s}_{U}, t}-a_{\mathbf{s}_{L}, t}
\end{aligned}
$$

or

$$
\begin{aligned}
\frac{X_{\mathbf{S}_{U}, t}-X_{\mathbf{s}_{L}, t}}{2} & =\frac{\theta_{U}-\theta_{L}}{2}+\sum_{i=1}^{p} \phi_{i} \frac{X_{\mathbf{s}_{U}, t-i}-X_{\mathbf{s}_{L}, t-i}}{2}+\sum_{i=0}^{p} \psi_{i} \frac{\omega_{L} X_{\mathbf{s}_{L}, t-i}-\omega_{U} X_{\mathbf{s}_{U}, t-i}}{2} \\
& +\frac{a_{\mathbf{s}_{U}, t}-a_{\mathbf{s}_{U}, t}}{2} .
\end{aligned}
$$

Therefore, this expression may be written as

$$
\begin{aligned}
R_{t} & =\frac{\theta_{U}-\theta_{L}}{2}+\sum_{i=1}^{p} \phi_{i} R_{t-i}+\sum_{i=0}^{p} \frac{\psi_{i}}{2}\left(\omega_{L}\left(C_{t-i}-R_{t-i}\right)-\omega_{U}\left(C_{t-i}+R_{t-i}\right)\right) \\
& +\frac{a_{\mathbf{s}_{U}, t}-a_{\mathbf{s}_{L}, t}}{2} \\
& =\frac{\theta_{U}-\theta_{L}}{2}+\sum_{i=1}^{p} \phi_{i} R_{t-i}-\sum_{i=0}^{p} \psi_{i}\left(\frac{\omega_{U}-\omega_{L}}{2} C_{t-i}+\frac{\omega_{L}+\omega_{U}}{2} R_{t-i}\right) \\
& +\frac{a_{\mathbf{s}_{U}, t}-a_{\mathbf{s}_{L}, t}}{2}
\end{aligned}
$$




$$
\begin{aligned}
& =\frac{\theta_{U}-\theta_{L}}{2}-\psi_{0} \frac{\omega_{L}+\omega_{U}}{2} R_{t}+\sum_{i=1}^{p}\left(\phi_{i}-\psi_{i} \frac{\omega_{L}+\omega_{U}}{2}\right) R_{t-i} \\
& +\sum_{i=0}^{p} \psi_{i}\left(-\frac{\omega_{U}-\omega_{L}}{2}\right) C_{t-i}+\frac{a_{\mathbf{s}_{U}, t}-a_{\mathbf{s}_{L}, t}}{2} \\
\Leftrightarrow R_{t} & =\frac{\theta_{U}-\theta_{L}}{2+\psi_{0}\left(\omega_{L}+\omega_{U}\right)}+\sum_{i=1}^{p} \frac{\phi_{i}-\psi_{i} \frac{\omega_{L}+\omega_{U}}{2}}{1+\psi_{0} \frac{\omega_{L}+\omega_{U}}{2}} R_{t-i} \\
& +\sum_{i=0}^{p} \frac{-\psi_{i} \frac{\omega_{U}-\omega_{L}}{2}}{1+\psi_{0} \frac{\omega_{L}+\omega_{U}}{2}} C_{t-i}+\frac{a_{\mathbf{s}_{U}, t}-a_{\mathbf{s}_{L}, t}}{2+\psi_{0}\left(\omega_{L}+\omega_{U}\right)} \\
& =\pi_{R}+\sum_{i=0}^{p} \pi_{R, i}^{C} C_{t-i}+\sum_{i=1}^{p} \pi_{R, i}^{R} R_{t-i}+\eta_{R_{t}}
\end{aligned}
$$

where, assuming $\psi_{0} \neq-2 /\left(\omega_{L}+\omega_{U}\right)$,

$$
\begin{aligned}
\pi_{R} & =\frac{\theta_{U}-\theta_{L}}{2+\psi_{0}\left(\omega_{L}+\omega_{U}\right)} ; \quad \pi_{R, i}^{C}=\frac{-\psi_{i} \frac{\omega_{U}-\omega_{L}}{2}}{1+\psi_{0} \frac{\omega_{L}+\omega_{U}}{2}} \quad i=0, \ldots, p ; \\
\pi_{R, i}^{R}= & \frac{\phi_{i}-\psi_{i} \frac{\omega_{L}+\omega_{U}}{2}}{1+\psi_{0} \frac{\omega_{L}+\omega_{U}}{2}} \quad i=1, \ldots, p ; \quad \eta_{R_{t}}=\frac{a_{\mathbf{s}_{U}, t}-a_{\mathbf{s}_{L}, t}}{2+\psi_{0}\left(\omega_{L}+\omega_{U}\right)}
\end{aligned}
$$

and $\eta_{R_{t}}$ is an independent white noise process with mean and variance

$$
\begin{aligned}
& E\left(\eta_{R_{t}}\right)=E\left(\frac{a_{\mathbf{s}_{U}, t}-a_{\mathbf{s}_{L}, t}}{2+\psi_{0}\left(\omega_{L}+\omega_{U}\right)}\right)=0 \\
& V\left(\eta_{R_{t}}\right)=\sigma_{\eta_{R}}^{2}=V\left(\frac{a_{\mathbf{s}_{U}, t}-a_{\mathbf{S}_{L}, t}}{2+\psi_{0}\left(\omega_{L}+\omega_{U}\right)}\right)=\frac{\sigma_{a_{L}}^{2}+\sigma_{a_{U}}^{2}-2 \sigma_{a_{L U}}}{\left(2+\psi_{0}\left(\omega_{L}+\omega_{U}\right)\right)^{2}} .
\end{aligned}
$$

Joining equations (12) and (15), we conclude that $\mathbf{Y}_{t}$ follows the $\operatorname{SVAR}(p)$ model

$$
\Delta \mathbf{Y}_{t}=\mathbf{\Pi}+\sum_{i=1}^{p} \boldsymbol{\Pi}_{i} \mathbf{Y}_{t-i}+\mathbf{V}_{t}
$$

where $\mathbf{V}_{t}=\left(\eta_{C_{t}}, \eta_{R_{t}}\right)^{T}$ is a vector independent white noise with $\eta_{C_{t}}$ and $\eta_{R_{t}}$ given above such that

$$
\begin{gathered}
\operatorname{Cov}\left(\eta_{C_{t}}, \eta_{R_{t+k}}\right)=E\left(\eta_{C_{t}} \eta_{R_{t+k}}\right)=\left\{\begin{array}{cc}
E\left(\frac{a_{\mathbf{s}_{U}, t}^{2}-a_{\mathbf{s}_{L}, t}^{2}}{4-\psi_{0}^{2}\left(\omega_{L}+\omega_{U}\right)^{2}}\right) & \text { if } k=0 \\
0 & \text { if } k \neq 0
\end{array}\right. \\
=\left\{\begin{array}{cc}
\frac{\sigma_{a_{U}}^{2}-\sigma_{a_{L}}^{2}}{4-\psi_{0}^{2}\left(\omega_{L}+\omega_{U}\right)^{2}} & \text { if } k=0 \\
0 & \text { if } k \neq 0
\end{array}=\left\{\begin{array}{cc}
\sigma_{\eta_{C R}} & \text { if } k=0 \\
0 & \text { if } k \neq 0
\end{array}\right.\right.
\end{gathered}
$$


and

$$
\begin{aligned}
\boldsymbol{\Delta} & =\left(\begin{array}{cc}
1 & -\pi_{C, 0}^{R} \\
-\pi_{R, 0}^{C} & 1
\end{array}\right) \\
\boldsymbol{\Pi} & =\left(\pi_{C}, \pi_{R}\right)^{T} \\
\mathbf{\Pi}_{i} & =\left(\begin{array}{cc}
\pi_{C, i}^{C} & \pi_{C, i}^{R} \\
\pi_{R, i}^{C} & \pi_{R, i}^{R}
\end{array}\right) \quad i=1, \ldots, p
\end{aligned}
$$

with the parameters $\pi_{C}, \pi_{C, i}^{C}$ and $\pi_{C, i}^{R}$ given in (13) and the parameters $\pi_{R}, \pi_{R, i}^{C}$ and $\pi_{R, i}^{R}$ given in (16), for $i=0, \ldots, p$. The matrix $\boldsymbol{\Delta}$ has to be nonsingular, requiring that $\pi_{R, 0}^{C} \neq-1 / \pi_{C, 0}^{R}$ or, in terms of the parameters of model (11),

$$
\phi_{0} \psi_{0} \frac{\omega_{U}-\omega_{L}}{2}+\psi_{0}^{2} \frac{\omega_{L}^{2}-\omega_{U}^{2}}{4} \neq 1-\psi_{0}^{2}\left(\frac{\omega_{L}+\omega_{U}}{2}\right)^{2} .
$$

We note that, as shown above, when $\mathbf{X}_{t}$ follows a $\operatorname{STAR}(p)$ model, $\mathbf{Y}_{t}$ does not follow a $\operatorname{STAR}(p)$, but a $\operatorname{SVAR}(p)$ model (Lütkepohl and Krätzig, 2004), which differs from the situation when $\mathbf{X}_{t}$ is a $\operatorname{VAR}(p)$ model. In fact, in the latter case, both $\mathbf{X}_{t}$ and $\mathbf{Y}_{t}$ follow $\operatorname{VAR}(p)$ models.

It is also important to analyze two particular cases. The first is that of a symmetric matrix $\mathbf{W}$ in model (8), i.e., when $\omega_{L}=\omega_{U}=\omega$ in model (11), which occurs in the general case. In such situation, the parameters in (12) and (15) given in (13) and (16) respectively become

$$
\begin{aligned}
& \pi_{C}=\frac{\theta_{L}+\theta_{U}}{2\left(1-\omega \psi_{0}\right)} ; \quad \pi_{C, i}^{C}=\frac{\phi_{i}+\omega \psi_{i}}{1-\omega \psi_{0}} \quad i=1, \ldots, p ; \quad \pi_{C, i}^{R}=0 \quad i=0, \ldots, p \\
& \pi_{R}=\frac{\theta_{U}-\theta_{L}}{2\left(1+\omega \psi_{0}\right)} ; \quad \pi_{R, i}^{R}=\frac{\phi_{i}-\omega \psi_{i}}{1+\omega \psi_{0}} \quad i=1, \ldots, p ; \quad \pi_{R, i}^{C}=0 \quad i=0, \ldots, p
\end{aligned}
$$

and $\eta_{C_{t}}=\frac{a_{\mathbf{s}_{L}, t}+a_{\mathbf{s}_{U}, t}}{2-2 \omega \psi_{0}}$ and $\eta_{R_{t}}=\frac{a_{\mathbf{s}_{U}, t}-a_{\mathbf{s}_{L}, t}}{2+2 \omega \psi_{0}}$ with, from (14), (17) and (19),

$$
\begin{aligned}
& E\left(\eta_{C_{t}}\right)=0 \\
& V\left(\eta_{C_{t}}\right)=\sigma_{\eta_{C}}^{2}=\frac{\sigma_{a_{L}}^{2}+\sigma_{a_{U}}^{2}+2 \sigma_{a_{L U}}}{4\left(1-\omega \psi_{0}\right)^{2}} \\
& E\left(\eta_{R_{t}}\right)=0 \\
& V\left(\eta_{R_{t}}\right)=\sigma_{\eta_{R}}^{2}=\frac{\sigma_{a_{L}}^{2}+\sigma_{a_{U}}^{2}-2 \sigma_{a_{L U}}}{4\left(1+\omega \psi_{0}\right)^{2}}
\end{aligned}
$$




$$
\operatorname{Cov}\left(\eta_{C_{t}}, \eta_{R_{t+k}}\right)=\left\{\begin{array}{cl}
\sigma_{\eta_{C R}} & \text { if } k=0 \\
0 & \text { if } k \neq 0
\end{array}=\left\{\begin{array}{cl}
\frac{\sigma_{a_{U}}^{2}-\sigma_{a_{L}}^{2}}{4\left(1-\omega^{2} \psi_{0}^{2}\right)} & \text { if } k=0 \\
0 & \text { if } k \neq 0
\end{array}\right.\right.
$$

assuming $\psi_{0} \neq \pm 1 / \omega$.

The second case is when the weights are standardized to sum up to 1. In such situation, $\omega_{L}=\omega_{U}=\omega=1$ and the parameters in (20) and (21) become

$$
\begin{aligned}
& \pi_{C}=\frac{\theta_{L}+\theta_{U}}{2\left(1-\psi_{0}\right)} ; \quad \pi_{C, i}^{C}=\frac{\phi_{i}+\psi_{i}}{1-\psi_{0}} \quad i=1, \ldots, p ; \quad \pi_{C, i}^{R}=0 \quad i=0, \ldots, p \\
& \pi_{R}=\frac{\theta_{U}-\theta_{L}}{2\left(1+\psi_{0}\right)} ; \quad \pi_{R, i}^{R}=\frac{\phi_{i}-\psi_{i}}{1+\psi_{0}} \quad i=1, \ldots, p ; \quad \pi_{R, i}^{C}=0 \quad i=0, \ldots, p
\end{aligned}
$$

and $\eta_{C_{t}}=\frac{a_{\mathbf{s}_{L}, t}+a_{\mathbf{s}_{U}, t}}{2\left(1-\psi_{0}\right)}$ and $\eta_{R_{t}}=\frac{a_{\mathbf{s}_{U}, t}-a_{\mathbf{s}_{L}, t}}{2\left(1+\psi_{0}\right)}$ with, from (22), (23) and (24),

$$
\begin{aligned}
E\left(\eta_{C_{t}}\right) & =0 \\
V\left(\eta_{C_{t}}\right) & =\sigma_{\eta_{C}}^{2}=\frac{\sigma_{a_{L}}^{2}+\sigma_{a_{U}}^{2}+2 \sigma_{a_{L U}}}{4\left(1-\psi_{0}\right)^{2}} \\
E\left(\eta_{R_{t}}\right) & =0 \\
V\left(\eta_{R_{t}}\right) & =\sigma_{\eta_{R}}^{2}=\frac{\sigma_{a_{L}}^{2}+\sigma_{a_{U}}^{2}-2 \sigma_{a_{L U}}}{4\left(1+\psi_{0}\right)^{2}} \\
\operatorname{Cov}\left(\eta_{C_{t}}, \eta_{R_{t+k}}\right) & =\left\{\begin{array}{cc}
\sigma_{\eta_{C R}} & \text { if } k=0 \\
0 & \text { if } k \neq 0
\end{array}=\left\{\begin{array}{cc}
\frac{\sigma_{a_{U}}^{2}-\sigma_{a_{L}}^{2}}{4\left(1-\psi_{0}^{2}\right)} & \text { if } k=0 \\
0 & \text { if } k \neq 0
\end{array}\right.\right.
\end{aligned}
$$

assuming $\psi_{0} \neq \pm 1$. Consequently, from (12) and (15), we conclude in both cases that $C_{t}$ and $R_{t}$ follow univariate $\operatorname{AR}(p)$ models:

$$
\begin{aligned}
& C_{t}=\pi_{C}+\sum_{i=1}^{p} \pi_{C, i}^{C} C_{t-i}+\eta_{C_{t}} \\
& R_{t}=\pi_{R}+\sum_{i=1}^{p} \pi_{R, i}^{R} R_{t-i}+\eta_{R_{t}} .
\end{aligned}
$$

As mentioned above, the $\operatorname{STAR}(p)$ model (11) can be used for prediction according to (10) with

$$
\widehat{\mathbf{X}}_{N}(m)=\left(\begin{array}{c}
\hat{X}_{\mathbf{s}_{L}, N}(m) \\
\hat{X}_{\mathbf{s}_{U}, N}(m)
\end{array}\right) ; \mathbf{I}_{2}-\psi_{0} \mathbf{W}=\left(\begin{array}{cc}
1 & -\psi_{0} \omega_{U} \\
-\psi_{0} \omega_{L} & 1
\end{array}\right) ; \boldsymbol{\Theta}=\left(\begin{array}{c}
\theta_{L} \\
\theta_{U}
\end{array}\right)
$$


$\phi_{i} \mathbf{I}_{2}+\psi_{i} \mathbf{W}=\left(\begin{array}{cc}\phi_{i} & \psi_{i} \omega_{U} \\ \psi_{i} \omega_{L} & \phi_{i}\end{array}\right) ; E_{N}\left(\mathbf{X}_{N+m-i}\right)=\left(\begin{array}{c}E_{N}\left(X_{\mathbf{s}_{L}, N+m-i}\right) \\ E_{N}\left(X_{\mathbf{s}_{U}, N+m-i}\right)\end{array}\right) i=1, \ldots, p$

assuming that $1-\psi_{0}^{2} \omega_{L} \omega_{U} \neq 0 \Leftrightarrow \psi_{0} \neq \pm 1 / \sqrt{\omega_{L} \omega_{U}}$ so that $\mathbf{I}_{2}-\psi_{0} \mathbf{W}$ is nonsingular. If $\omega_{L}=\omega_{U}=\omega$, then $\psi_{0} \neq \pm 1 / \omega$ which has been assumed above in (20) to (24). If $\omega=1$, then $\psi_{0} \neq \pm 1$, assumed in (25) to (29).

Similarly, given the data $\mathbf{Y}_{1}, \ldots, \mathbf{Y}_{N}$ and letting $E_{N}(\cdot)=E\left(\cdot \mid \mathbf{Y}_{N}, \mathbf{Y}_{N-1}, \ldots\right)$, the minimum mean square error predictor of $\mathbf{Y}_{N+m}(m=1,2, \ldots)$ is, from (18),

$$
\begin{aligned}
\widehat{\mathbf{Y}}_{N}(m) & =E_{N}\left(\mathbf{Y}_{N+m}\right)=\boldsymbol{\Delta}^{-1}\left(\boldsymbol{\Pi}+\sum_{i=1}^{p} \boldsymbol{\Pi}_{i} E_{N}\left(\mathbf{Y}_{N+m-i}\right)\right) \\
& =\boldsymbol{\Delta}^{-1}\left(\boldsymbol{\Pi}+\sum_{i=1}^{p} \boldsymbol{\Pi}_{i} \widehat{\mathbf{Y}}_{N}(m-i)\right)
\end{aligned}
$$

where

$$
E_{N}\left(\mathbf{Y}_{N+m-i}\right)= \begin{cases}\widehat{\mathbf{Y}}_{N}(m-i) & m-i>0 \\ \mathbf{Y}_{N+m-i} & m-i \leq 0\end{cases}
$$

Since the conditional expectation is a linear operator, it is possible to predict $\mathbf{X}_{N+m}$ from $\widehat{\mathbf{Y}}_{N}(m)$ :

$$
\begin{aligned}
\widehat{X}_{\mathbf{s}_{L}, N}(m) & =E\left(X_{\mathbf{s}_{L}, N+m} \mid\left(X_{\mathbf{s}_{L}, N}, X_{\mathbf{s}_{U}, N}\right)^{T},\left(X_{\mathbf{s}_{L}, N-1}, X_{\mathbf{s}_{U}, N-1}\right)^{T}, \ldots\right) \\
& =E\left(C_{N+m}-R_{N+m} \mid\left(C_{N}, R_{N}\right)^{T},\left(C_{N-1}, R_{N-1}\right)^{T}, \ldots\right) \\
& =E\left(C_{N+m} \mid\left(C_{N}, R_{N}\right)^{T},\left(C_{N-1}, R_{N-1}\right)^{T}, \ldots\right) \\
& -E\left(R_{N+m} \mid\left(C_{N}, R_{N}\right)^{T},\left(C_{N-1}, R_{N-1}\right)^{T}, \ldots\right) \\
& =\widehat{C}_{N}(m)-\widehat{R}_{N}(m)
\end{aligned}
$$

and

$$
\begin{aligned}
\widehat{X}_{\mathbf{S}_{U}, N}(m) & =E\left(X_{\mathbf{s}_{U}, N+m} \mid\left(X_{\mathbf{s}_{L}, N}, X_{\mathbf{S}_{U}, N}\right)^{T},\left(X_{\mathbf{S}_{L}, N-1}, X_{\mathbf{S}_{U}, N-1}\right)^{T}, \ldots\right) \\
& =E\left(C_{N+m}+R_{N+m} \mid\left(C_{N}, R_{N}\right)^{T},\left(C_{N-1}, R_{N-1}\right)^{T}, \ldots\right) \\
& =\widehat{C}_{N}(m)+\widehat{R}_{N}(m) .
\end{aligned}
$$

Similarly, it is also possible to predict $\mathbf{Y}_{N+m}$ from $\widehat{\mathbf{X}}_{N}(m)$ :

$$
\widehat{C}_{N}(m)=E\left(C_{N+m} \mid\left(C_{N}, R_{N}\right)^{T},\left(C_{N-1}, R_{N-1}\right)^{T}, \ldots\right)
$$




$$
\begin{aligned}
& =E\left(\frac{X_{\mathbf{s}_{L}, N}+X_{\mathbf{s}_{U}, N}}{2} \mid\left(X_{\mathbf{s}_{L}, N}, X_{\mathbf{s}_{U}, N}\right)^{T},\left(X_{\mathbf{s}_{L}, N-1}, X_{\mathbf{s}_{U}, N-1}\right)^{T}, \ldots\right) \\
& =\frac{1}{2}\left[E\left(X_{\mathbf{s}_{L}, N+m} \mid\left(X_{\mathbf{s}_{L}, N}, X_{\mathbf{s}_{U}, N}\right)^{T},\left(X_{\mathbf{s}_{L}, N-1}, X_{\mathbf{s}_{U}, N-1}\right)^{T}, \ldots\right)\right. \\
& \left.+E\left(X_{\mathbf{s}_{U}, N+m} \mid\left(X_{\mathbf{s}_{L}, N}, X_{\mathbf{s}_{U}, N}\right)^{T},\left(X_{\mathbf{s}_{L}, N-1}, X_{\mathbf{s}_{U}, N-1}\right)^{T}, \ldots\right)\right] \\
& =\frac{\widehat{X}_{\mathbf{s}_{L}, N}(m)+\widehat{X}_{\mathbf{s}_{U}, N}(m)}{2}
\end{aligned}
$$

and

$$
\begin{aligned}
\widehat{R}_{N}(m) & =E\left(R_{N+m} \mid\left(C_{N}, R_{N}\right)^{T},\left(C_{N-1}, R_{N-1}\right)^{T}, \ldots\right) \\
& =E\left(\frac{X_{\mathbf{s}_{U}, N}-X_{\mathbf{s}_{L}, N}}{2} \mid\left(X_{\mathbf{s}_{U}, N}, X_{\mathbf{s}_{L}, N}\right)^{T},\left(X_{\mathbf{s}_{L}, N-1}, X_{\mathbf{s}_{U}, N-1}\right)^{T}, \ldots\right) \\
& =\frac{\widehat{X}_{\mathbf{s}_{U}, N}(m)-\widehat{X}_{\mathbf{s}_{L}, N}(m)}{2} .
\end{aligned}
$$

Prediction with the $\operatorname{STAR}(p)$ model (11) requires that the predicted bounds satisfy $\hat{X}_{\mathbf{s}_{L}, t}(m) \leq \hat{X}_{\mathbf{s}_{U}, t}(m)$ for every period $t$. Similarly, prediction with the $\operatorname{SVAR}(p)$ model (18) requires that the predicted values of the radius are positive, $\widehat{R}_{t}(m) \geq 0$ for every $t$. Therefore, in both cases it is required that $\hat{X}_{\mathbf{S}_{U}, t}(m)-\hat{X}_{\mathbf{s}_{L}, t}(m) \geq 0$ for which sufficient conditions on the parameters are derived next. We note this is a similar approach to that of conditional heteroscedastic ARCH and GARCH models of Engle (1982) and Bollerslev (1986) to ensure positiveness of the conditional variance (see also Tsay, 2005).

From model (11), the interval range is

$$
\begin{aligned}
X_{\mathbf{s}_{U}, t}-X_{\mathbf{s}_{L}, t} & =\theta_{U}-\theta_{L}+\sum_{i=1}^{p} \phi_{i}\left(X_{\mathbf{s}_{U}, t-i}-X_{\mathbf{s}_{L}, t-i}\right)+\sum_{i=0}^{p} \psi_{i}\left(\omega_{L} X_{\mathbf{s}_{L}, t-i}-\omega_{U} X_{\mathbf{s}_{U}, t-i}\right) \\
& +a_{\mathbf{s}_{U}, t}-a_{\mathbf{s}_{L}, t} .
\end{aligned}
$$

For prediction purposes, the white noise processes $a_{\mathbf{s}_{L}, t}$ and $a_{\mathbf{s}_{U}, t}$ are set to zero (their expected values) and consequently it is required that, for every $t$,

$$
\theta_{U}-\theta_{L}+\sum_{i=1}^{p} \phi_{i}\left(X_{\mathbf{s}_{U}, t-i}-X_{\mathbf{s}_{L}, t-i}\right)+\sum_{i=0}^{p} \psi_{i}\left(\omega_{L} X_{\mathbf{s}_{L}, t-i}-\omega_{U} X_{\mathbf{s}_{U}, t-i}\right) \geq 0 .
$$

For this condition to hold, it is sufficient that

$$
\begin{aligned}
& \theta_{U} \geq \theta_{L}, \\
& \phi_{i} \geq 0 \text { since } X_{\mathbf{s}_{U}, t-i}-X_{\mathbf{s}_{L}, t-i} \geq 0 \quad(i=1, \ldots, p)
\end{aligned}
$$


and, for $(i=0, \ldots, p)$,

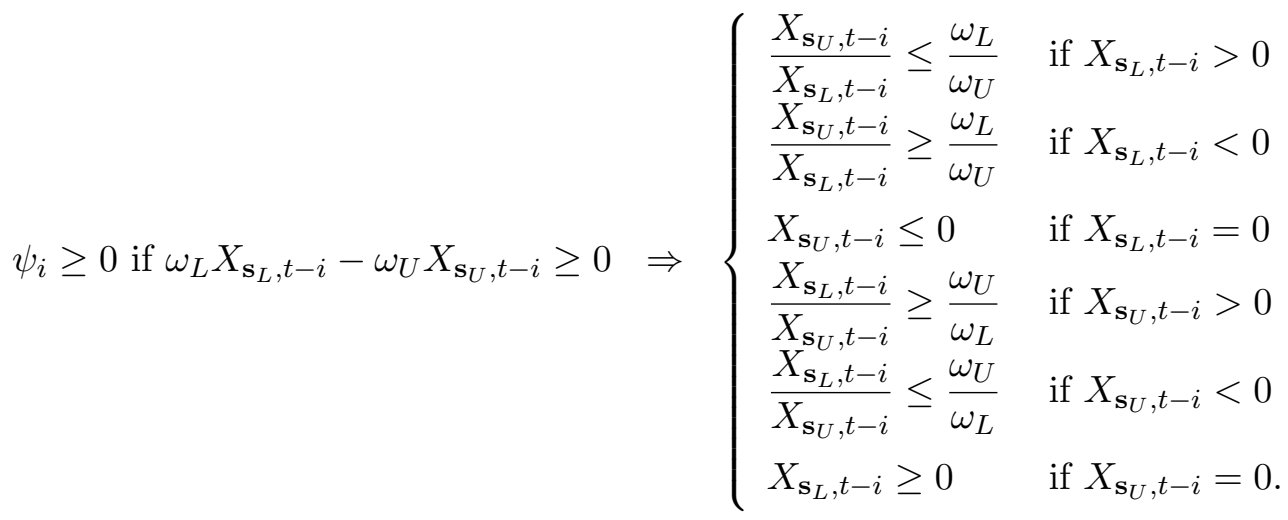

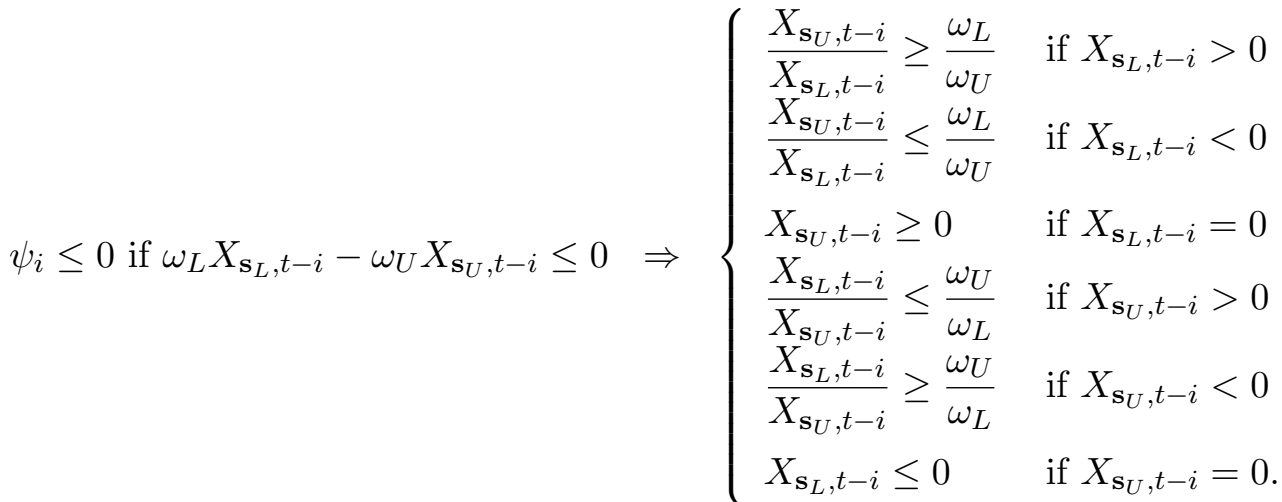

If at least one of the above conditions (36) to (39) does not hold, then it is not possible to derive sufficient conditions and only (35) is required.

Concerning the radius $R_{t}$ in equation (15), it is required that

$$
\pi_{R}+\sum_{i=0}^{p} \pi_{R, i}^{C} C_{t-i}+\sum_{i=1}^{p} \pi_{R, i}^{R} R_{t-i} \geq 0
$$

for every $t$, since the white noise process is also set to zero (its expected value) for prediction. Assuming first that $C_{t} \geq 0$ for every $t$ (which is true if $X_{\mathbf{s}_{U}, t} \geq\left|X_{\mathbf{s}_{L}, t}\right|$ ), it is sufficient for (40) that $\pi_{R} \geq 0, \pi_{R, i}^{C} \geq 0(i=0,1, \ldots, p)$ and $\pi_{R, i}^{R} \geq 0(i=1, \ldots, p)$, since $R_{t-i} \geq 0, i=1, \ldots, p$ i. These conditions impose the following restrictions on the parameters of the $\operatorname{STAR}(p)$ model (11):

$$
\begin{aligned}
& \pi_{R}=\frac{\theta_{U}-\theta_{L}}{2+\psi_{0}\left(\omega_{L}+\omega_{U}\right)} \geq 0 \Rightarrow \theta_{U} \geq \theta_{L} \text { and } \psi_{0}>-\frac{2}{\omega_{L}+\omega_{U}} \quad \text { (recall that } \theta_{U}<\theta_{L} \text { is not } \\
& \text { possible because of (36)). }
\end{aligned}
$$

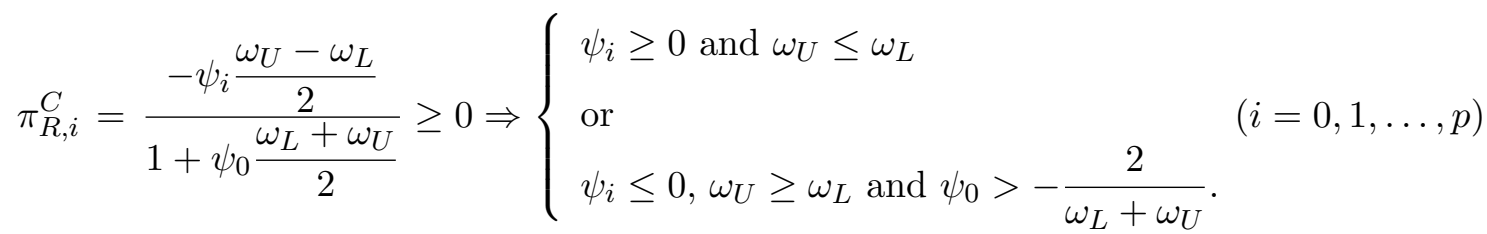


Note that $\psi_{i} \leq 0, \omega_{U} \leq \omega_{L}$ and $\psi_{0}<-\frac{2}{\omega_{L}+\omega_{U}}$ is not valid because, although it satisfies $\pi_{R, i}^{C} \geq 0$, the condition on $\psi_{0}$ violates (41).

$\pi_{R, i}^{R}=\frac{\phi_{i}-\psi_{i} \frac{\omega_{L}+\omega_{U}}{2}}{1+\psi_{0} \frac{\omega_{L}+\omega_{U}}{2}} \geq 0 \Rightarrow \phi_{i} \geq \psi_{i} \frac{\omega_{L}+\omega_{U}}{2}$ and $\psi_{0}>-\frac{2}{\omega_{L}+\omega_{U}} \quad(i=1, \ldots, p)$.

Note that $\phi_{i} \leq \psi_{i} \frac{\omega_{L}+\omega_{U}}{2}$ and $\psi_{0}<-\frac{2}{\omega_{L}+\omega_{U}}$ is not valid because, although it satisfies $\pi_{R, i}^{R} \geq 0$, the condition on $\psi_{0}$ violates (41).

Assuming now that $C_{t} \leq 0$ for every $t$ (which is true if $X_{\mathbf{s}_{U}, t} \leq\left|X_{\mathbf{s}_{L}, t}\right|$ ), it is sufficient for (40) that $\pi_{R} \geq 0, \pi_{R, i}^{C} \leq 0(i=0,1, \ldots, p)$ and $\pi_{R, i}^{R} \geq 0(i=1, \ldots, p)$. The conditions on $\pi_{R}$ and $\pi_{R, i}^{R}$ are the same as above and thus lead again to (41) and (43) respectively, the new restrictions on the parameters of the $\operatorname{STAR}(p)$ model (11) are as follows:

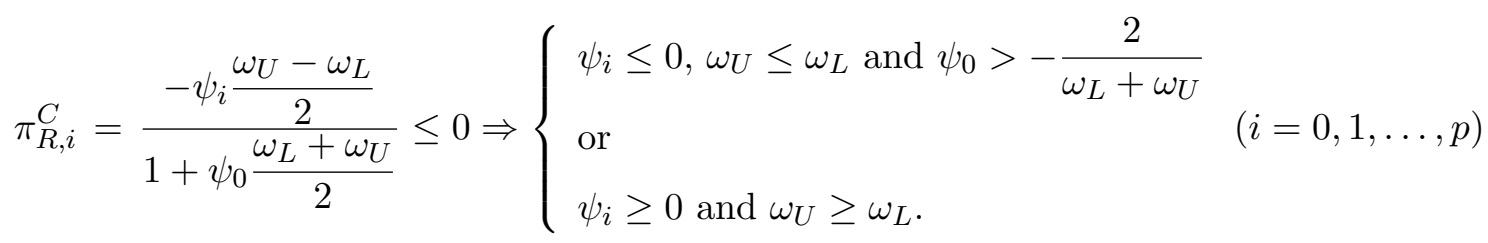

Note that $\psi_{i} \leq 0, \omega_{U} \geq \omega_{L}$ and $\psi_{0}<-\frac{2}{\omega_{L}+\omega_{U}}$ is not valid because, although it satisfies $\pi_{R, i}^{C} \geq 0$, the condition on $\psi_{0}$ violates (41).

If at least one of the above conditions (41), (42) or (43) and (43) does not hold, then it is not possible to derive sufficient conditions and only (40) is required.

If the matrix $\mathbf{W}$ is symmetric, i.e., $\omega_{L}=\omega_{U}=\omega$, new sufficient conditions can be derived instead of (36) to (39) or of (41) to (43). In fact, concerning the former, (34) is

$$
\begin{aligned}
X_{\mathbf{s}_{U}, t}-X_{\mathbf{s}_{L}, t} & =\theta_{U}-\theta_{L}+\sum_{i=1}^{p} \phi_{i}\left(X_{\mathbf{s}_{U}, t-i}-X_{\mathbf{s}_{L}, t-i}\right)+\sum_{i=0}^{p} \omega \psi_{i}\left(X_{\mathbf{s}_{L}, t-i}-X_{\mathbf{s}_{U}, t-i}\right) \\
& +a_{\mathbf{s}_{U}, t}-a_{\mathbf{s}_{L}, t} \\
& =\theta_{U}-\theta_{L}-\omega \psi_{0}\left(X_{\mathbf{s}_{U}, t}-X_{\mathbf{s}_{L}, t}\right)+\sum_{i=1}^{p}\left(\phi_{i}-\omega \psi_{i}\right)\left(X_{\mathbf{s}_{U}, t-i}-X_{\mathbf{s}_{L}, t-i}\right) \\
& +a_{\mathbf{s}_{U}, t}-a_{\mathbf{s}_{L}, t} \\
\Leftrightarrow X_{\mathbf{s}_{U}, t}-X_{\mathbf{s}_{L}, t} & =\frac{\theta_{U}-\theta_{L}}{1+\omega \psi_{0}}+\frac{1}{1+\omega \psi_{0}} \sum_{i=1}^{p}\left(\phi_{i}-\omega \psi_{i}\right)\left(X_{\mathbf{s}_{U}, t-i}-X_{\mathbf{s}_{L}, t-i}\right) \\
& +\frac{a_{\mathbf{s}_{U}, t}-a_{\mathbf{s}_{L}, t}}{1+\omega \psi_{0}} .
\end{aligned}
$$


Dropping the white noise process in the right-hand side of this equation, condition (35) is now replaced by

$$
\frac{\theta_{U}-\theta_{L}}{1+\omega \psi_{0}}+\frac{1}{1+\omega \psi_{0}} \sum_{i=1}^{p}\left(\phi_{i}-\omega \psi_{i}\right)\left(X_{\mathbf{s}_{U}, t-i}-X_{\mathbf{s}_{L}, t-i}\right) \geq 0
$$

and the sufficient conditions are as follows.

$$
\begin{aligned}
\theta_{U} & \geq \theta_{L}, \psi_{0}>-\frac{1}{\omega} \text { and } \phi_{i} \geq \omega \psi_{i}(i=1, \ldots, p) \text { or } \\
\theta_{U} & \leq \theta_{L}, \psi_{0}<-\frac{1}{\omega} \text { and } \phi_{i} \leq \omega \psi_{i}(i=1, \ldots, p) .
\end{aligned}
$$

Concerning the model of the radius (31) with parameters given by (21), the conditions are as follows (recall that these conditions are the same when $C_{t} \geq 0$ for every $t$ and when $C_{t} \leq 0$ for every $\left.t\right)$ :

$$
\begin{gathered}
\pi_{R}=\frac{\theta_{U}-\theta_{L}}{2\left(1+\omega \psi_{0}\right)} \geq 0 \Rightarrow\left\{\begin{array}{l}
\theta_{U} \geq \theta_{L} \text { and } \psi_{0}>-\frac{1}{\omega} \\
\text { or } \\
\theta_{U} \leq \theta_{L} \text { and } \psi_{0}<-\frac{1}{\omega}
\end{array}\right. \\
\pi_{R, i}^{R}=\frac{\phi_{i}-\omega \psi_{i}}{1+\omega \psi_{0}} \geq 0 \Rightarrow\left\{\begin{array}{l}
\phi_{i} \geq \omega \psi_{i} \text { and } \psi_{0}>-\frac{1}{\omega} \\
\text { or } \\
\phi_{i} \leq \omega \psi_{i} \text { and } \psi_{0}<-\frac{1}{\omega} .
\end{array}(i=1, \ldots, p)\right.
\end{gathered}
$$

It is important to recall that these conditions are also valid when $\omega=1$ (standardized weights).

In practice, the above conditions have to be verified by the parameter estimates ensuring that the predicted values of the upper bound are not less than those of the lower bound and that the predicted values of the radius are not negative.

Thus, realizing that a $\operatorname{STAR}(p)$ model is appropriate for an ITS leads to the conclusion that its center and radius do not follow a bivariate STAR model, but an $\operatorname{SVAR}(p)$. When the weight matrix is symmetric, i.e., when $\omega_{L}=\omega_{U}=\omega$ or when these weights are standardized to sum $1\left(\omega_{L}=\omega_{U}=1\right)$, the center and the radius follow univariate $\operatorname{AR}(p)$ models each. Finally, the interval bounds may be predicted from their $\operatorname{STAR}(p)$ model and the center and radius may be predicted from their $\operatorname{SVAR}(p)$. It is also possible to predict the former from the latter and vice-versa. Sufficient conditions on the model parameters were derived 
in order to ensure that the upper bound predicted value is not smaller than that of the lower bound, or, equivalently, that the predicted value of the radius is not negative.

\section{Comparing predictive accuracy through simulation}

In order to compare the predictive performance of the models discussed above, we conducted two simulation experiments where 1,000 time series of 6,010 observations each were generated from different $\operatorname{STAR}(p)$ models with $p=1, \ldots, 6$.

In the first experiment, the data were generated from (8) with parameter values given below and the minimum and the maximum at each time period were retained forming the lower and upper bounds of the interval time series respectively. The first six models considered are STAR processes with $n=4$, weight matrix $\mathbf{W}$ (this matrix is standardized so that all rows add up to one), residual covariance matrix $\boldsymbol{\Sigma}_{a}$ and vector of intercepts $\boldsymbol{\Theta}$, $\mathbf{W}=\left(\begin{array}{cccc}0.000 & 0.333 & 0.133 & 0.533 \\ 0.233 & 0.000 & 0.419 & 0.349 \\ 0.148 & 0.667 & 0.000 & 0.185 \\ 0.444 & 0.417 & 0.139 & 0.000\end{array}\right) ; \boldsymbol{\Sigma}_{a}=\left(\begin{array}{cccc}0.11 & 0.10 & 0.05 & 0.01 \\ 0.10 & 0.20 & 0.07 & 0.03 \\ 0.05 & 0.07 & 0.05 & 0.04 \\ 0.01 & 0.03 & 0.04 & 0.08\end{array}\right) ; \boldsymbol{\Theta}=\left(\begin{array}{c}1 \\ 0.5 \\ 0.3 \\ 0.8\end{array}\right)$.

The autoregressive parameters are as follows.

Model $1-\operatorname{STAR}(1)$ with $\psi_{0}=-0.6, \psi_{1}=0.1, \phi_{1}=0.2$.

Model $2-\operatorname{STAR}(2)$ with $\psi_{0}=0.3, \psi_{1}=0.2, \psi_{2}=-0.25, \phi_{1}=0.4, \phi_{2}=-0.2$.

Model $3-\operatorname{STAR}(3)$ with $\psi_{0}=0.5, \psi_{1}=0.2, \psi_{2}=-0.2, \psi_{3}=0.05, \phi_{1}=0.25, \phi_{2}=$ $-0.15, \phi_{3}=0.1$.

Model $4-\operatorname{STAR}(4)$ with $\psi_{0}=0.4, \psi_{1}=-0.2, \psi_{2}=-0.17, \psi_{3}=0.05, \psi_{4}=0.02, \phi_{1}=$ $-0.15, \phi_{2}=-0.1, \phi_{3}=0.08, \phi_{4}=0.04$.

Model $5-\operatorname{STAR}(5)$ with $\psi_{0}=0.5, \psi_{1}=-0.25, \psi_{2}=-0.2, \psi_{3}=-0.12, \psi_{4}=$ $0.05, \psi_{5}=0.03, \phi_{1}=-0.2, \phi_{2}=-0.15, \phi_{3}=0.1, \phi_{4}=0.08, \phi_{5}=0.05$.

Model $6-\operatorname{STAR}(6)$ with $\psi_{0}=-0.6, \psi_{1}=0.1, \psi_{2}=0.1, \psi_{3}=-0.12, \psi_{4}=0.08, \psi_{5}=$ $0.05, \psi_{6}=-0.04, \phi_{1}=0.2, \phi_{2}=0.15, \phi_{3}=-0.1, \phi_{4}=0.1, \phi_{5}=0.08, \phi_{6}=-0.03$. 
We also considered six STAR processes with $n=5$, standardized weight matrix, residual covariance matrix and vector of intercepts,

$$
\mathbf{W}=\left(\begin{array}{ccccc}
0.000 & 0.270 & 0.108 & 0.432 & 0.189 \\
0.182 & 0.000 & 0.327 & 0.273 & 0.218 \\
0.114 & 0.514 & 0.000 & 0.143 & 0.229 \\
0.340 & 0.319 & 0.106 & 0.000 & 0.234 \\
0.184 & 0.316 & 0.211 & 0.289 & 0.000
\end{array}\right) ; \boldsymbol{\Sigma}_{a}=\left(\begin{array}{ccccc}
0.80 & 0.60 & 0.40 & 0.25 & 0.70 \\
0.60 & 1.20 & 0.35 & 0.40 & 0.80 \\
0.40 & 0.35 & 0.55 & 0.25 & 0.30 \\
0.25 & 0.40 & 0.25 & 0.40 & 0.30 \\
0.70 & 0.80 & 0.30 & 0.30 & 1.50
\end{array}\right) ;
$$

$\Theta^{T}=\left(\begin{array}{lllll}1 & 0.5 & 0.3 & 0.8 & 0.6\end{array}\right)$.

The autoregressive parameters are as follows.

Model $7-\operatorname{STAR}(1)$ with $\psi_{0}=-0.6, \psi_{1}=0.1, \phi_{1}=0.3$.

Model $8-\operatorname{STAR}(2)$ with $\psi_{0}=-0.6, \psi_{1}=0.2, \psi_{2}=0.1, \phi_{1}=0.3, \phi_{2}=0.2$.

Model $9-\operatorname{STAR}(3)$ with $\psi_{0}=0.5, \psi_{1}=0.2, \psi_{2}=-0.2, \psi_{3}=0.05, \phi_{1}=0.25, \phi_{2}=$ $-0.15, \phi_{3}=0.1$.

Model $10-\operatorname{STAR}(4)$ with $\psi_{0}=-0.7, \psi_{1}=-0.25, \psi_{2}=-0.2, \psi_{3}=0.05, \psi_{4}=$ $0.02, \phi_{1}=-0.2, \phi_{2}=-0.15, \phi_{3}=0.1, \phi_{4}=0.08$.

Model $11-\operatorname{STAR}(5)$ with $\psi_{0}=0.5, \psi_{1}=-0.25, \psi_{2}=-0.2, \psi_{3}=-0.12, \psi_{4}=$ $0.05, \psi_{5}=0.03, \phi_{1}=-0.2, \phi_{2}=-0.15, \phi_{3}=0.1, \phi_{4}=0.08, \phi_{5}=0.05$.

Model $12-\operatorname{STAR}(6)$ with $\psi_{0}=-0.45, \psi_{1}=0.1, \psi_{2}=0.1, \psi_{3}=-0.12, \psi_{4}=$ $0.08, \psi_{5}=0.05, \psi_{6}=-0.04, \phi_{1}=0.2, \phi_{2}=0.15, \phi_{3}=-0.1, \phi_{4}=0.1, \phi_{5}=0.08, \phi_{6}=$ -0.03 .

In the second experiment, the interval bounds were generated from (11) with parameter values given below and a single standardized weight $\omega$, i.e., $\omega_{L}=\omega_{U}=\omega=1$, since this is a bivariate model. The residual covariance matrix $\boldsymbol{\Sigma}_{a}$ and the vector of intercepts $\boldsymbol{\Theta}$ are

$$
\boldsymbol{\Sigma}_{a}=\left(\begin{array}{cc}
0.5 & 0.6 \\
0.6 & 0.75
\end{array}\right) ; \boldsymbol{\Theta}=\left(\begin{array}{c}
0.8 \\
0.4
\end{array}\right)
$$

The autoregressive parameters are as follows. 
Model $13-\operatorname{STAR}(1)$ with $\psi_{0}=0.5, \psi_{1}=-0.3, \phi_{1}=0.6$.

Model $14-\operatorname{STAR}(2)$ with $\psi_{0}=-0.6, \psi_{1}=0.5, \psi_{2}=0.1, \phi_{1}=0.6, \phi_{2}=0.2$.

Model $15-\operatorname{STAR}(3)$ with $\psi_{0}=-0.6, \psi_{1}=0.3, \psi_{2}=-0.25, \psi_{3}=0.05, \phi_{1}=0.4, \phi_{2}=$ $-0.2, \phi_{3}=0.1$.

Model $16-\operatorname{STAR}(4)$ with $\psi_{0}=-0.5, \psi_{1}=0.2, \psi_{2}=0.1, \psi_{3}=-0.1, \psi_{4}=-0.07$, $\phi_{1}=0.25, \phi_{2}=0.2, \phi_{3}=-0.1, \phi_{4}=-0.05$.

Model $17-\operatorname{STAR}(5)$ with $\psi_{0}=-0.65, \psi_{1}=0.25, \psi_{2}=0.1, \psi_{3}=-0.1, \psi_{4}=$ $-0.08, \psi_{5}=0.02, \phi_{1}=0.3, \phi_{2}=0.15, \phi_{3}=0.1, \phi_{4}=-0.08, \phi_{5}=0.05$.

Model $18-\operatorname{STAR}(6)$ with $\psi_{0}=-0.45, \psi_{1}=0.1, \psi_{2}=0.1, \psi_{3}=-0.12, \psi_{4}=$ $0.08, \psi_{5}=0.05, \psi_{6}=-0.04, \phi_{1}=0.2, \phi_{2}=0.15, \phi_{3}=-0.1, \phi_{4}=0.1, \phi_{5}=0.08, \phi_{6}=$ -0.03 .

In both experiments, the last 10 observations were left out from the data for an assessment of the models' out-of-sample predictive performance. For each time series, model (11) was estimated by three-stage least squares (we considered a symmetric matrix $\mathbf{W}$ with a single standardized weight $\omega$, i.e., $\omega_{L}=\omega_{U}=\omega=1$, since this is a bivariate model) and the VAR model (2) (Arroyo, 2008; García-Ascanio and Maté, 2009) and the univariate AR models of the center and radius (Maia, De Carvalho and Ludemir, 2008) were estimated by ordinary least squares or maximum likelihood.

The 10 out-of-sample observations of the interval time series were then predicted with the fitted models (the predictions of the center and radius of the univariate AR approach were used to compute those of the interval bounds). The accuracy of those predictions was measured by the interval bounds' Mean Absolute Error (MAE) and Root Mean Square Error (RMSE) and by computing interval distance measures between the actual and the predicted values (since the data have an interval form). To this purpose, different measures are available in the literature and we will consider three interval distance measures: the Hausdorff distance, the interval Euclidean distance and the interval City-Block distance.

Let $I_{i}=\left[l_{i}, u_{i}\right]$ and $I_{j}=\left[l_{j}, u_{j}\right]$ two intervals to be compared. The Hausdorff distance, the interval Euclidean distance and the interval City-Block distance between $I_{i}$ and $I_{j}$ are 
respectively

$$
\begin{aligned}
d_{H}\left(I_{i}, I_{j}\right) & =\max \left\{\left\{\left|l_{i}-l_{j}\right|,\left|u_{i}-u_{j}\right|\right\}\right. \\
d_{2}\left(I_{i}, I_{j}\right) & =\sqrt{\left(l_{i}-l_{j}\right)^{2}+\left(u_{i}-u_{j}\right)^{2}} \\
d_{1}\left(I_{i}, I_{j}\right) & =\left|l_{i}-l_{j}\right|+\left|u_{i}-u_{j}\right| .
\end{aligned}
$$

The Hausdorff distance between two sets is the maximum distance of a set to the nearest point in the other set, i.e., two sets are close in terms of the Hausdorff distance if every point of either set is close to some point of the other set. Interval Euclidean and City-Block distances are just the counterparts of the corresponding distances for real values; if we embed the interval set in $\mathbb{R}^{2}$, where one dimension is used for the lower and the other for the upper bound of the intervals, then these distances are just the Euclidean and City-Block distances between the corresponding points in the two-dimensional space.

Table 1 (in the Appendix) shows the values of the accuracy measures for the different models considered in both experiments and for the three approaches, namely the STAR model (11), the VAR model (2) and the univariate AR models of the center and radius, denoted by STAR, VAR and AR respectively. The results show that none of the approaches outperforms the others, since their predictive accuracy is always extremely close or even coincident. It is also interesting to note that the predictive accuracy is better for models with $\psi_{0}<0$, i.e., when the interval bounds are contemporaneously related in the opposite direction. Many other STAR models were tried as data-generating processes in the two experiments with similar results.

\section{Application}

As a practical application, we consider the Irish wind data of Haslett and Raftery (1989), available at Statlib (http://lib.stat.cmu.edu/datasets/), which consists of time series of daily average wind speed at eleven synoptic metereological stations in Ireland in the period 19611978. Detailed information on this dataset can be found in Haslett and Raftery (1989), in Gneiting, Genton and Guttorp (2007) and in the references therein. From the eleven stations, we selected five: Birr, Dublin, Kilkenny, Shannon and Valentia. The observations for February 29 were removed for a better estimation of the seasonal trend component. The year of 1978 was left out from the dataset for an out-of-sample evaluation of predictive performance and therefore only the 1961-1977 data will be modeled. Following the authors 
mentioned above, the square root transform was first applied to the time series of daily average wind speed stabilizing the variance over both stations and time periods and bringing the marginal distributions closer to normality. The seasonal effect was estimated by calculating the average of the square root of the daily means over all years and stations for each day of the year and then regressing the results on a small set of annual harmonics. Subtraction of the estimated seasonal effect from the square root of the daily means yields deseasonalized data. The station-specific means were also removed and the result will be referred to as the time series of velocity measures. The same transformations were applied to the year of 1978 with the seasonal trend component and the station-specific means estimated from the 1961-1977 period.

The minimum and the maximum of the daily velocity measures at the five selected stations are respectively the lower and upper bounds of an interval time series to which the bivariate $\operatorname{STAR}(p)$ model (11) was fitted. We consider a symmetric matrix $\mathbf{W}$ with a single standardized weight $\omega$, i.e., $\omega_{L}=\omega_{U}=\omega=1$, since this is a bivariate model. The order of the model, i.e., the value of $p$, was determined by starting with a large value, estimating the parameters, applying tests sequentially and decreasing that order until a suitable model is found. We started with $p=8$ and selected $p=6$, since the estimated parameters for both $p=7$ and $p=8$ were not statistically significant. Order selection criteria were also used (Lütkepohl and Krätzig, 2004) such as the Akaike Information Criterion (AIC), the Hannan-Quinn Criterion (HQC) and the Schwarz Criterion (SC) and they suggest $p=3$, but the model diagnostic checking showed a better fit with $p=6$. Therefore, model (11) with $p=6$ was estimated by three-stage least squares and the results are given in table 2 .

Model diagnostic checking shows a satisfactory fit. The estimated parameters up to lag 3 are significant and, although $\hat{\phi}_{4}, \hat{\phi}_{5}, \hat{\psi}_{4}$ and $\hat{\psi}_{5}$ are not, we decided to keep $p=6$ because $\hat{\phi}_{6}$ and $\hat{\psi}_{6}$ are clearly significant (5\% significance level). Moreover, as mentioned above, model selection criteria such as AIC, HQC and SC suggest $p=3$ (because of the lack of significance of the parameters at lags 4 and 5) but, even though this would still be acceptable, $p=6$ shows a much better fit. In fact, the residuals of both equations $\hat{a}_{\mathbf{s}_{L}, t}$ and $\hat{a}_{\mathbf{s}_{U}, t}$ are approximately white noise, since their plots show no clear defficiencies and their correlograms show very few significant autocorrelations that do not give rise to concern because they occur only occasionally and at very high lags. The p-values of the portmanteau 
Table 2. Estimation results of the STAR(6) model of the ITS

\begin{tabular}{cccccccc}
\hline Parameter & Estimate & t-stat & p-value & Parameter & Estimate & t-stat & p-value \\
\hline$\theta_{L}$ & -0.0862 & -4.6495 & 0.0000 & $\psi_{0}$ & -0.5741 & -5.4209 & 0.0000 \\
$\theta_{U}$ & 0.0726 & 3.8018 & 0.0001 & $\psi_{1}$ & 0.3989 & 8.7124 & 0.0000 \\
$\phi_{1}$ & 0.4952 & 24.5542 & 0.0000 & $\psi_{2}$ & -0.0654 & -7.1079 & 0.0000 \\
$\phi_{2}$ & -0.0482 & -3.6194 & 0.0003 & $\psi_{3}$ & 0.0265 & 2.6464 & 0.0081 \\
$\phi_{3}$ & 0.0379 & 3.8799 & 0.0001 & $\psi_{4}$ & 0.0073 & 0.7747 & 0.4385 \\
$\phi_{4}$ & 0.0131 & 1.3363 & 0.1815 & $\psi_{5}$ & -0.0148 & -1.5845 & 0.1131 \\
$\phi_{5}$ & -0.0030 & -0.2908 & 0.7712 & $\psi_{6}$ & 0.0186 & 2.1327 & 0.0330 \\
$\phi_{6}$ & 0.0282 & 3.0617 & 0.0022 & & & & \\
\hline
\end{tabular}

$\hat{\sigma}_{a_{L}}^{2}=0.4619, \hat{\sigma}_{a_{U}}^{2}=0.4229, \hat{\sigma}_{a_{L U}}=0.4364$

Ljung-Box statistic at lag 25 are respectively 0.764 and 0.897 , which is not significant. The residuals are also approximately Normal, as shown by their histograms and by the JarqueBera test whose p-values are respectively 0.132 and 0.053 , both nonsignificant at the $5 \%$ level (the latter value is not far from being significant, but this could be expected because of the deviations from normality of the original data). The residual cross-correlations are also generally nonsignificant at nonzero lags. Thus, we may conclude that this model is adequate for the data. We also note that a $\operatorname{VAR}(6)$ model would require a much larger number of parameters than the $\operatorname{STAR}(6)$ model above, i.e., the latter provides a good fit and is much more parsimonious. Futhermore, it also shows the contemporaneous relationship between the interval bounds, as given by $\hat{\psi}_{0}=-0.5741$ (table 2), which is an important feature of an interval time series that the other approaches cannot uncover.

We can now derive the models for the center and radius of the ITS, given by (30) and (31) respectively, since $\hat{\psi}_{0}=-0.5741 \neq \pm 1$ as required. Both are $\operatorname{AR}(6)$ models with their parameters given by $(25)$ and (26) whose estimates, given in table 3, are computed from the estimation results of the STAR(6) model given in table 2. From (27) and (28), the estimated residual variances are respectively $\hat{\sigma}_{\eta_{C}}^{2}=0.1773$ and $\hat{\sigma}_{\eta_{R}}^{2}=0.0165$.

The estimated STAR(6) model above was next used for prediction of the velocity measures. For this purpose, we first check if the parameter estimates given in table 2 satisfy 
Table 3. Estimation results of the AR models of the center and radius

\begin{tabular}{cccc}
\hline Parameter & Estimate & Parameter & Estimate \\
\hline$\pi_{C}$ & -0.0043 & $\pi_{R}$ & 0.1864 \\
$\pi_{C, 1}^{C}$ & 0.5680 & $\pi_{R, 1}^{R}$ & 0.2261 \\
$\pi_{C, 2}^{C}$ & -0.0722 & $\pi_{R, 2}^{R}$ & 0.0404 \\
$\pi_{C, 3}^{C}$ & 0.0409 & $\pi_{R, 3}^{R}$ & 0.0268 \\
$\pi_{C, 4}^{C}$ & 0.0130 & $\pi_{R, 4}^{R}$ & 0.0136 \\
$\pi_{C, 5}^{C}$ & -0.0113 & $\pi_{R, 5}^{R}$ & 0.0277 \\
$\pi_{C, 6}^{C}$ & 0.0297 & $\pi_{R, 6}^{R}$ & 0.0225 \\
\hline
\end{tabular}

the conditions ensuring the predicted value of the upper bound is not smaller than that of the lower bound and that the predicted radius is not negative. Since $\omega_{L}=\omega_{U}=\omega=1$, the appropriate condition on the STAR(6) model parameter estimates is (46) or (47); it is clear that the former is satisfied which also implies that conditions (48) and (49) concerning the model of the radius are also satisfied (in fact, the parameter estimates of this model given in table 3 are all positive).

We considered 10-day forecasts in several periods covering the 12 months of the year of 1978 , the test period. The data before each forecasting period were subject to the same procedure described above for the years 1961 to 1977 and the model was re-estimated (the estimates were extremely stable, i.e., they showed only minor changes everytime the model was re-estimated). To assess the forecasting performance, table 4 shows the mean absolute error (MAE), the root mean square error (RMSE) and the mean and the standard deviation of the three interval distance measures (Hausdorff, Euclidean and City-Block distances) between the actual and the forecast intervals for each 10-day period and for the whole of the 1978 forecasts. The forecasting performance is generally satisfactory. In fact, it is similar to and is often better than that obtained by Gneiting, Genton and Guttorp (2007) for 1-day forecasts at each station, while we have 10-day forecasts of the minimum and maximum of 5 stations which is more difficult to predict and therefore favors our results. The upper bound forecasts generally outperform those of the lower bound, since they have a smaller MAE and RMSE most of the months as well as for the whole test period. Center and 
radius forecasts were also computed from their respective $\mathrm{AR}(6)$ models (table 3 ) and used to obtain the forecasts of the upper and lower bounds as mentioned above. As expected, the results were coincident, i.e., predicting the upper and lower bounds from their $\operatorname{STAR}(6)$ model or from the corresponding $\mathrm{AR}(6)$ models of the center and radius leads to the same forecasts.

Table 4. Predictive Performance of the STAR Model

\begin{tabular}{|c|c|c|c|c|c|c|c|}
\hline \multirow[b]{2}{*}{ Month } & \multirow[b]{2}{*}{ Days } & \multicolumn{4}{|c|}{ MAE } & \multicolumn{2}{|c|}{ RMSE } \\
\hline & & & $X_{\mathbf{s}_{L}}$ & $X_{\mathbf{s}_{U}}$ & & $X_{\mathbf{s}_{L}}$ & $X_{\mathbf{s}_{U}}$ \\
\hline January & $1-10$ & & 0.433 & 0.321 & & 0.510 & 0.408 \\
\hline February & $19-28$ & & 0.401 & 0.299 & & 0.520 & 0.394 \\
\hline March & $1-10$ & & 0.498 & 0.360 & & 0.605 & 0.456 \\
\hline April & $21-30$ & & 0.259 & 0.366 & & 0.323 & 0.416 \\
\hline May & $1-10$ & & 0.373 & 0.415 & & 0.440 & 0.484 \\
\hline June & $21-30$ & & 0.342 & 0.230 & & 0.388 & 0.288 \\
\hline July & $21-30$ & & 0.463 & 0.277 & & 0.516 & 0.359 \\
\hline August & $11-20$ & & 0.224 & 0.241 & & 0.279 & 0.296 \\
\hline September & $16-25$ & & 0.516 & 0.294 & & 0.634 & 0.351 \\
\hline October & $16-25$ & & 0.390 & 0.351 & & 0.440 & 0.384 \\
\hline November & $16-25$ & & 0.240 & 0.278 & & 0.280 & 0.311 \\
\hline December & $21-30$ & & 0.448 & 0.371 & & 0.514 & 0.418 \\
\hline \multirow[t]{2}{*}{ Total } & & & 0.382 & 0.317 & & 0.468 & 0.385 \\
\hline & & \multicolumn{2}{|c|}{ Hausdorff } & \multicolumn{2}{|c|}{ Euclidean } & \multicolumn{2}{|c|}{ City-Block } \\
\hline Month & Days & Mean & Std. dev. & Mean & Std. dev. & Mean & Std. dev. \\
\hline January & $1-10$ & 0.443 & 0.288 & 0.548 & 0.374 & 0.754 & 0.535 \\
\hline February & $19-28$ & 0.480 & 0.307 & 0.540 & 0.384 & 0.701 & 0.548 \\
\hline March & $1-10$ & 0.540 & 0.326 & 0.644 & 0.422 & 0.859 & 0.605 \\
\hline April & $21-30$ & 0.373 & 0.205 & 0.461 & 0.268 & 0.625 & 0.381 \\
\hline May & $1-10$ & 0.438 & 0.271 & 0.563 & 0.352 & 0.788 & 0.497 \\
\hline June & $21-30$ & 0.356 & 0.187 & 0.425 & 0.242 & 0.572 & 0.349 \\
\hline
\end{tabular}


Table 4 (cont.). Predictive Performance of the STAR Model

\begin{tabular}{lccccccc}
\hline & \multicolumn{3}{c}{ Hausdorff } & \multicolumn{2}{c}{ Euclidean } & \multicolumn{2}{c}{ City-Block } \\
\hline \multicolumn{1}{c}{ Month } & Days & Mean & Std. dev. & Mean & Std. dev. & Mean & Std. dev. \\
\hline July & $21-30$ & 0.471 & 0.231 & 0.555 & 0.310 & 0.739 & 0.458 \\
August & $11-20$ & 0.278 & 0.184 & 0.340 & 0.234 & 0.466 & 0.332 \\
September & $16-25$ & 0.527 & 0.377 & 0.602 & 0.426 & 0.810 & 0.578 \\
October & $16-25$ & 0.456 & 0.189 & 0.545 & 0.221 & 0.741 & 0.311 \\
November & $16-25$ & 0.302 & 0.140 & 0.375 & 0.197 & 0.518 & 0.286 \\
December & $21-30$ & 0.533 & 0.206 & 0.625 & 0.232 & 0.819 & 0.325 \\
\hline \multicolumn{1}{c}{ Total } & & 0.433 & 0.254 & 0.519 & 0.315 & 0.699 & 0.443 \\
\hline
\end{tabular}

Finally, the predictive accuracy of the other two approaches considered was also computed for comparison purposes. Therefore, a VAR $(p)$ model (Arroyo, 2008; García-Ascanio and Maté, 2009) was first fitted to the interval bounds and used to forecast. Following the same steps as for the STAR model above (order identification, estimation with OLS and diagnostic checking), a VAR(2) model seemed appropriate for the interval time series. Futhermore, univariate autoregressive models (Maia, De Carvalho and Ludermir, 2008) were also fitted to the center and radius, respectively $\operatorname{AR}(6)$ and $\operatorname{AR}(5)$ processes, and used to obtain forecasts of the upper and lower bounds as mentioned above. The predictive accuracy of the two approaches is given in table 5 . The comparison of tables 4 and 5 shows that the predictive accuracy of the STAR model and of the univariate AR models are extremely similar, almost coincident, which could be expected because the latter can be derived from the former, as previously shown. Moreover, they outperform the VAR model since, with very few exceptions and even though the differences are not large, the latter generally yields less accurate forecasts.

It is important to recall that, unlike the other approaches, STAR models also display the contemporaneous relationship at time period $t$ between the interval bounds (measured by the parameter $\left.\psi_{0}\right)$ which is an important aspect in interval time series analysis. 
Table 5. Predictive Performance of the VAR Model and of the Univariate AR Models

\begin{tabular}{|c|c|c|c|c|c|c|c|c|c|}
\hline \multirow[b]{3}{*}{ Month } & \multirow[b]{3}{*}{ Days } & \multicolumn{4}{|c|}{ MAE } & \multicolumn{4}{|c|}{ RMSE } \\
\hline & & \multicolumn{2}{|c|}{ VAR } & \multicolumn{2}{|c|}{$\mathrm{AR}$} & \multicolumn{2}{|c|}{ VAR } & \multicolumn{2}{|c|}{$\mathrm{AR}$} \\
\hline & & $X_{\mathbf{s}_{L}}$ & $X_{\mathbf{s}_{U}}$ & $X_{\mathbf{s}_{L}}$ & $X_{\mathbf{s}_{U}}$ & $X_{\mathbf{s}_{L}}$ & $X_{\mathbf{s}_{U}}$ & $X_{\mathbf{s}_{L}}$ & $X_{\mathbf{s}_{U}}$ \\
\hline January & $1-10$ & 0.448 & 0.339 & 0.434 & 0.319 & 0.524 & 0.424 & 0.511 & 0.406 \\
\hline February & $19-28$ & 0.411 & 0.312 & 0.401 & 0.299 & 0.530 & 0.406 & 0.519 & 0.392 \\
\hline March & $1-10$ & 0.510 & 0.357 & 0.497 & 0.361 & 0.618 & 0.463 & 0.604 & 0.458 \\
\hline April & $21-30$ & 0.280 & 0.377 & 0.261 & 0.363 & 0.329 & 0.426 & 0.323 & 0.414 \\
\hline May & $1-10$ & 0.381 & 0.419 & 0.376 & 0.415 & 0.441 & 0.483 & 0.442 & 0.484 \\
\hline June & $21-30$ & 0.340 & 0.214 & 0.342 & 0.233 & 0.382 & 0.276 & 0.387 & 0.291 \\
\hline July & $21-30$ & 0.465 & 0.279 & 0.463 & 0.276 & 0.517 & 0.361 & 0.516 & 0.358 \\
\hline August & $11-20$ & 0.240 & 0.246 & 0.230 & 0.234 & 0.291 & 0.301 & 0.286 & 0.291 \\
\hline September & $16-25$ & 0.493 & 0.276 & 0.516 & 0.291 & 0.601 & 0.323 & 0.636 & 0.349 \\
\hline October & $16-25$ & 0.411 & 0.370 & 0.392 & 0.350 & 0.456 & 0.400 & 0.442 & 0.383 \\
\hline November & $16-25$ & 0.246 & 0.294 & 0.237 & 0.280 & 0.306 & 0.339 & 0.275 & 0.315 \\
\hline December & $21-30$ & 0.454 & 0.354 & 0.446 & 0.371 & 0.515 & 0.400 & 0.514 & 0.416 \\
\hline \multirow[t]{3}{*}{ Total } & & 0.390 & 0.320 & 0.383 & 0.316 & 0.472 & 0.388 & 0.469 & 0.384 \\
\hline & & \multicolumn{4}{|c|}{ Hausdorff Distance } & \multicolumn{4}{|c|}{ Euclidean Distance } \\
\hline & & \multicolumn{2}{|c|}{ VAR } & \multicolumn{2}{|c|}{$\mathrm{AR}$} & \multicolumn{2}{|c|}{ VAR } & \multicolumn{2}{|c|}{$\mathrm{AR}$} \\
\hline & & & Std. & & Std. & & Std. & & Std. \\
\hline Month & Days & Mean & Dev. & Mean & Dev. & Mean & Dev. & Mean & Dev. \\
\hline January & $1-10$ & 0.458 & 0.291 & 0.444 & 0.289 & 0.570 & 0.380 & 0.548 & 0.374 \\
\hline February & $19-28$ & 0.490 & 0.322 & 0.479 & 0.306 & 0.556 & 0.390 & 0.539 & 0.384 \\
\hline March & $1-10$ & 0.546 & 0.337 & 0.539 & 0.326 & 0.652 & 0.436 & 0.643 & 0.423 \\
\hline April & $21-30$ & 0.392 & 0.197 & 0.375 & 0.201 & 0.483 & 0.251 & 0.460 & 0.267 \\
\hline May & $1-10$ & 0.445 & 0.259 & 0.439 & 0.271 & 0.571 & 0.337 & 0.564 & 0.352 \\
\hline June & $21-30$ & 0.353 & 0.179 & 0.355 & 0.186 & 0.419 & 0.229 & 0.426 & 0.242 \\
\hline July & $21-30$ & 0.473 & 0.228 & 0.471 & 0.232 & 0.559 & 0.307 & 0.555 & 0.310 \\
\hline August & $11-20$ & 0.292 & 0.179 & 0.281 & 0.184 & 0.356 & 0.232 & 0.342 & 0.236 \\
\hline
\end{tabular}


Table 5 (cont.). Predictive Performance of the VAR Model and of the Univariate AR Models

\begin{tabular}{ccccccccccc}
\hline & & \multicolumn{3}{c}{ Hausdorff Distance } & \multicolumn{3}{c}{ Euclidean Distance } \\
\hline & & \multicolumn{2}{c}{ VAR } & \multicolumn{2}{c}{ AR } & \multicolumn{2}{c}{ VAR } & AR \\
\hline \multirow{2}{*}{ Month } & Days & Mean & Dev. & Mean & Dev. & Mean & Dev. & Mean & Dev. \\
\hline September & $16-25$ & 0.504 & 0.350 & 0.527 & 0.379 & 0.576 & 0.387 & 0.600 & 0.429 \\
October & $16-25$ & 0.478 & 0.176 & 0.458 & 0.191 & 0.573 & 0.208 & 0.547 & 0.221 \\
November & $16-25$ & 0.311 & 0.186 & 0.302 & 0.144 & 0.390 & 0.250 & 0.374 & 0.198 \\
December & $21-30$ & 0.535 & 0.189 & 0.531 & 0.210 & 0.619 & 0.214 & 0.623 & 0.235 \\
\hline Total & & 0.440 & 0.251 & 0.433 & 0.255 & 0.527 & 0.310 & 0.518 & 0.315 \\
\hline
\end{tabular}

City-Block Distance

\begin{tabular}{lrrrrr}
\hline & & \multicolumn{2}{c}{ VAR } & \multicolumn{2}{c}{ AR } \\
\hline \multirow{1}{*}{ Month } & Days & Mean & Dev. & Mean & Dev. \\
\hline January & $1-10$ & 0.787 & 0.542 & 0.753 & 0.536 \\
February & $19-28$ & 0.724 & 0.547 & 0.699 & 0.547 \\
March & $1-10$ & 0.866 & 0.629 & 0.858 & 0.605 \\
April & $21-30$ & 0.657 & 0.357 & 0.625 & 0.381 \\
May & $1-10$ & 0.800 & 0.475 & 0.791 & 0.495 \\
June & $21-30$ & 0.554 & 0.332 & 0.575 & 0.348 \\
July & $21-30$ & 0.745 & 0.454 & 0.739 & 0.458 \\
August & $11-20$ & 0.486 & 0.331 & 0.465 & 0.335 \\
September & $16-25$ & 0.769 & 0.521 & 0.807 & 0.583 \\
October & $16-25$ & 0.780 & 0.298 & 0.741 & 0.311 \\
November & $16-25$ & 0.540 & 0.358 & 0.517 & 0.285 \\
December & $21-30$ & 0.808 & 0.301 & 0.817 & 0.329 \\
\hline \multicolumn{1}{c}{ Total } & & 0.710 & 0.437 & 0.699 & 0.444 \\
\hline
\end{tabular}




\section{Concluding remarks}

When interval-valued symbolic data are collected as an ordered sequence through time, they form an interval time series (ITS). In this paper, we proposed to model an ITS with a Space-time autoregressive process $\operatorname{STAR}(p)$, which appears to be appropriate for such data, given the link between the lower and upper bounds of the observed intervals. Based on this model for the ITS bounds we also showed that its center and radius follow a bivariate Structural Vector Autoregressive process $\operatorname{SVAR}(p)$ whose parameters are functions of those in the $\operatorname{STAR}(p)$ model; two especially relevant particular cases were analyzed. Concerning prediction, we derived the minimum mean square error predictor and sufficient conditions on the parameters to ensure that the predicted upper bound is not less than the lower bound or that the predicted values of the radius are not negative. A simulation study showed that the predictive accuracy of the different approaches discussed is very similar and that none of them outperforms the others. Finally, these models were applied to an ITS of the Irish wind speed and 10-day forecasts were computed showing good accuracy. We may thus conclude that modeling an interval time series with a Space-time Autoregressive process is appropriate and provides accurate forecasts.

\section{References}

Antunes, A.M.C. and Subba Rao, T. (2006). On hypotheses testing for the selection of spatio-temporal models, Journal of Time Series Analysis 27, 767-791.

Arroyo, J. (2008). Métodos de predicción para series temporales de intervalos e histogramas, Unpublished Ph.D. Dissertation, Universidad Pontificia Comillas, Madrid.

Arroyo, J., González-Rivera, G. and Maté, C. (2010). Forecasting with interval and histogram data. Some financial applications In: Ullah, A. and Giles, D., Balakrishnan, N., Schucany, W. and Schilling, E., eds. Handbook of Empirical Economics and Finance,(forthcoming). New York: Chapman and Hall/CR.

Bertrand, P. and Goupil, F. (2000). Descriptive statistics for symbolic data. In: Bock, H.H. and Diday, E., eds.,Analysis of Symbolic Data. Exploratory Methods for Extracting Statistical Information from Complex Data. Heidelberg: Springer, 106-124. 
Billard, L. and Diday, E. (2000). Regression analysis for interval-valued data. In: Kiers, H.A.L. and Rasson, J.P., eds. Data Analysis, Classification, and Related Methods, Proceedings IFCS'2000, Namur. Heidelberg: Springer Verlag, 369-374.

Billard, L. and Diday, E. (2003). From the Statistics of Data to the Statistics of Knowledge: Symbolic Data Analysis, Journal of the American Statistical Association 98, 470-487.

Billard, L. and Diday, E. (2006). Symbolic Data Analysis: Conceptual Statistics and Data Analysis. Chichester: John Wiley and Sons.

Bock, H.-H. and Diday, E. (Eds.) (2000) Analysis of Symbolic Data, Exploratory Methods for Extracting Statistical Information from Complex Data. Heidelberg: Springer.

Bollerslev, T. (1986). Generalized autoregressive conditional heteroskedasticity, Journal of Econometrics 31, 307-327.

Box, G.E.P., Jenkins, G.M. and Reinsel G.C. (2008). Time Series Analysis: Forecasting and Control, 4th. ed. Hoboken, New Jersey: John Wiley and Sons.

Brito, P. (2007). Modelling and Analysing Interval Data. In: Decker, R., Lenz, H.-J., eds. Advances in Data Analysis. Berlin, Heidelberg, New-York: Springer, 197-208.

Brockwell, P.J., Davis, R.A. (2002). Introduction to Time Series and Forecasting. New York: Springer Verlag.

Chouakria, A., Cazes, P., Diday, E. (2000). Symbolic Principal Component Analysis. In: Bock, H.-H. and Diday, E., eds. Analysis of Symbolic Data. Exploratory Methods for Extracting Statistical Information from Complex Data. Heidelberg: Springer. 200212 .

Cliff, A.D. and Ord, J.K. (1975). Model building and the analysis of spatial pattern in human geography, Journal of the Royal Statistical Society B 37, 297-328.

Cressie, N.A.C. (1993). Statistics for Spatial Data. New York: John Wiley and Sons.

Diday, E. and Noirhomme, M. (Eds.) (2008). Symbolic Data and the SODAS Software. Chichester: John Wiley and Sons. 
Diggle, P.J. and Ribeiro, P.J. Jr. (2007). Model-based Geostatistics, Springer, New York.

Duarte Silva, A.P. and Brito, P. (2006). Linear discriminant analysis for interval data, Computational Statistics 21, 2, 289-308.

Engle, R. F. (1982). Autoregressive conditional heteroscedasticity with estimates of the variance of United Kingdom inflations, Econometrica 50, 987-1007.

Finkenstädt, B., Held, L. and Isham, V. (Eds.) (2007). Statistical Methods for SpatioTemporal Systems. London: Chapman and Hall/CRC.

García-Ascanio, C. and Maté, C. (2009). Electric power demand forecasting using interval time series: A comparison between VAR and iMLPC, Energy Policy 38, 715-725.

Gneiting, T., Genton, M.G. and Guttorp, P. (2007). Geostatistical space-time models, stationarity, separability, and full symmetry. In: Finkenstädt, B., Held, L. and Isham, V., eds. Statistical Methods for Spatio-Temporal Systems. London: Chapman and Hall/CRC, 151-175.

González-Rivera, G. and Arroyo, J. (2011). Time series modelling of histogram-valued data: The daily histogram time series of S\&P500 intradaily returns. International Journal of Forecasting 28(1), 20-33.

Han, A., Hong, Y., Lai, K. and Wang, S. (2008). Interval time series analysis with an application to the Sterling-Dollar exchange rate, Journal of Systems Science and Complexity 21(4), 558-573.

Haslett, J. and Raftery, A.E. (1989). Space-time modelling with long-memory dependence: assessing Ireland's wind-power resource (with discussion), Applied Statistics 38, 1, 150.

Johnston, J. and Dinardo, D. (1997). Econometric Methods, 4th. ed. New York: McGrawHill.

Lauro, C. and Palumbo, F. (2005). Principal component analysis for non-precise data. In: Vichi, M. et al eds. New Developments in Classification and Data Analysis. Berlin, Heidelberg: Springer, 173-184. 
Le, N.D. and Zidek, J.V. (2006). Statistical Analysis of Environmental Space-Time Processes. New York: Springer.

Lütkepohl, H. and Krätzig, M. (Eds.) (2004). Applied Time Series Econometrics, Cambridge University Press, New York.

Maia, A.L.S., De Carvalho, F.A.T. and Ludermir, T.D. (2008). Forecasting models for interval-valued time series, Neurocomputing 71 (16-18), 3344-3352.

Neto, E.A.L. and De Carvalho, F.A.T. (2008). Centre and range method for fitting a linear regression model to symbolic intervalar data, Computational Statistics $\& 3$ Data Analysis 52, 3, 1500-1515.

Neto, E.A.L. and De Carvalho, F.A.T. (2010). Constrained linear regression models for symbolic interval-valued variables, Computational Statistics 85 Data Analysis 54, 2, 333-347.

Noirhomme-Fraiture, M. and Brito, P. (2011). Far Beyond the Classical Data Models: Symbolic Data Analysis. Statistical Analysis and Data Mining 4, 2, 157-170.

Pfeifer, P. and Deutsch, S. (1980). A three stage interactive procedure for space-time modeling, Technometrics 22, 35-47.

Teles, P. and Brito, P. (2005). Modelling interval time series data, Proceedings of the 3rd IASC World Conference on Computational Statistics and Data Analysis, Limassol, Cyprus.

Tsay, R. (2005). Analysis of Financial Time Series, 2nd ed. Hoboken, New Jersey: John Wiley and Sons.

Wei, W.W.S. (2006). Time Series Analysis: Univariate and Multivariate Methods, 2nd. ed. New York: Addison-Wesley. 
Appendix 
Table 1. Predictive Performance of Different Approaches

\begin{tabular}{|c|c|c|c|c|c|c|}
\hline \multicolumn{7}{|c|}{ STAR processes with $n=4$} \\
\hline & \multicolumn{6}{|c|}{ MAE } \\
\hline & \multicolumn{2}{|c|}{ STAR } & \multicolumn{2}{|c|}{ VAR } & \multicolumn{2}{|c|}{$\mathrm{AR}$} \\
\hline Model & $X_{\mathbf{s}_{L}}$ & $X_{\mathbf{s}_{U}}$ & $X_{\mathbf{s}_{L}}$ & $X_{\mathbf{s}_{U}}$ & $X_{\mathbf{s}_{L}}$ & $X_{\mathbf{s}_{U}}$ \\
\hline Model 1 & 0.166 & 0.204 & 0.166 & 0.204 & 0.166 & 0.204 \\
\hline Model 2 & 0.425 & 0.423 & 0.423 & 0.419 & 0.423 & 0.419 \\
\hline Model 3 & 0.566 & 0.560 & 0.572 & 0.558 & 0.566 & 0.557 \\
\hline Model 4 & 0.466 & 0.452 & 0.467 & 0.447 & 0.462 & 0.446 \\
\hline Model 5 & 0.598 & 0.584 & 0.605 & 0.585 & 0.602 & 0.584 \\
\hline \multirow[t]{3}{*}{ Model 6} & 0.160 & 0.2 & 0.160 & 0.220 & 0.159 & 0.220 \\
\hline & \multicolumn{6}{|c|}{ RMSE } \\
\hline & \multicolumn{2}{|c|}{ STAR } & \multicolumn{2}{|c|}{ VAR } & \multicolumn{2}{|c|}{$\mathrm{AR}$} \\
\hline Model & $X_{\mathbf{s}_{L}}$ & $X_{\mathbf{s}_{U}}$ & $X_{\mathbf{s}_{L}}$ & $X_{\mathbf{s}_{U}}$ & $X_{\mathbf{s}_{L}}$ & $X_{\mathbf{s}_{U}}$ \\
\hline Model 1 & 0.210 & 0.250 & 0.209 & 0.250 & 0.209 & 0.249 \\
\hline Model 2 & 0.508 & 0.505 & 0.510 & 0.504 & 0.510 & 0.504 \\
\hline Model 3 & 0.663 & 0.657 & 0.693 & 0.677 & 0.687 & 0.676 \\
\hline Model 4 & 0.554 & 0.536 & 0.564 & 0.538 & 0.559 & 0.538 \\
\hline Model 5 & 0.686 & 0.672 & 0.730 & 0.706 & 0.728 & 0.705 \\
\hline \multirow[t]{2}{*}{ Model 6} & 0.200 & 0.270 & 0.200 & 0.269 & 0.200 & 0.269 \\
\hline & \multicolumn{3}{|c|}{ Hausdorff distance } & \multicolumn{3}{|c|}{ Euclidean distance } \\
\hline M & STAR & $\mathrm{VA}$ & $\mathrm{AR}$ & ST & VAR & $\mathrm{AR}$ \\
\hline Model 1 & 0.266 & 0.266 & 0.266 & 0.293 & 0.293 & 0.293 \\
\hline Model 2 & 0.497 & 0.494 & 0.494 & 0.616 & 0.612 & 0.612 \\
\hline Model 3 & 0.629 & 0.640 & 0.632 & 0.816 & 0.815 & 0.808 \\
\hline Model 4 & 0.526 & 0.531 & 0.526 & 0.642 & 0.663 & 0.658 \\
\hline Model 5 & 0.655 & 0.669 & 0.666 & 0.835 & 0.855 & 0.853 \\
\hline Model 6 & 0.277 & 0.276 & 0.276 & 0.303 & 0.302 & 0.302 \\
\hline
\end{tabular}


Table 1 (cont.). Predictive Performance of Different Approaches City-Block distance

\begin{tabular}{lccc}
\hline Model & STAR & VAR & AR \\
\hline Model 1 & 0.371 & 0.370 & 0.370 \\
Model 2 & 0.847 & 0.842 & 0.842 \\
Model 3 & 1.136 & 1.131 & 1.124 \\
Model 4 & 0.908 & 0.914 & 0.908 \\
Model 5 & 1.182 & 1.189 & 1.186 \\
Model 6 & 0.381 & 0.379 & 0.379 \\
\hline
\end{tabular}

STAR processes with $n=5$

\begin{tabular}{|c|c|c|c|c|c|c|}
\hline \multirow[b]{3}{*}{ Model } & \multicolumn{6}{|c|}{ MAE } \\
\hline & \multicolumn{2}{|c|}{ STAR } & \multicolumn{2}{|c|}{ VAR } & \multicolumn{2}{|c|}{$\mathrm{AR}$} \\
\hline & $X_{\mathbf{s}_{L}}$ & $X_{\mathbf{s}_{U}}$ & $X_{\mathbf{s}_{L}}$ & $X_{\mathbf{s}_{U}}$ & $X_{\mathbf{s}_{L}}$ & $X_{\mathbf{s}_{U}}$ \\
\hline Model 7 & 0.524 & 0.484 & 0.523 & 0.486 & 0.523 & 0.486 \\
\hline Model 8 & 0.543 & 0.512 & 0.546 & 0.516 & 0.546 & 0.516 \\
\hline Model 9 & 1.578 & 1.572 & 1.591 & 1.577 & 1.580 & 1.574 \\
\hline Model 10 & 0.505 & 0.479 & 0.507 & 0.479 & 0.507 & 0.479 \\
\hline Model 11 & 1.650 & 1.641 & 1.640 & 1.629 & 1.637 & 1.628 \\
\hline \multirow[t]{3}{*}{ Model 12} & 0.533 & 0.505 & 0.536 & 0.505 & 0.536 & 0.505 \\
\hline & \multicolumn{6}{|c|}{ RMSE } \\
\hline & \multicolumn{2}{|c|}{ STAR } & \multicolumn{2}{|c|}{ VAR } & \multicolumn{2}{|c|}{$\mathrm{AR}$} \\
\hline Model & $X_{\mathbf{s}_{L}}$ & $X_{\mathbf{s}_{U}}$ & $X_{\mathbf{s}_{L}}$ & $X_{\mathbf{s}_{U}}$ & $X_{\mathbf{s}_{L}}$ & $X_{\mathbf{s}_{U}}$ \\
\hline Model 7 & 0.625 & 0.588 & 0.642 & 0.598 & 0.642 & 0.598 \\
\hline Model 8 & 0.649 & 0.616 & 0.667 & 0.631 & 0.667 & 0.631 \\
\hline Model 9 & 1.869 & 1.856 & 1.928 & 1.906 & 1.916 & 1.904 \\
\hline Model 10 & 0.616 & 0.587 & 0.626 & 0.591 & 0.626 & 0.591 \\
\hline Model 11 & 1.988 & 1.978 & 1.976 & 1.964 & 1.972 & 1.963 \\
\hline Model 12 & 0.642 & 0.612 & 0.657 & 0.620 & 0.657 & 0.620 \\
\hline
\end{tabular}


Table 1 (cont.). Predictive Performance of Different Approaches

\begin{tabular}{|c|c|c|c|c|c|c|}
\hline & \multicolumn{3}{|c|}{ Hausdorff distance } & \multicolumn{3}{|c|}{ Euclidean distance } \\
\hline Model & STAR & VAR & $\mathrm{AR}$ & STAR & VAR & $\mathrm{AR}$ \\
\hline Model 7 & 0.704 & 0.714 & 0.714 & 0.764 & 0.793 & 0.793 \\
\hline Model 8 & 0.726 & 0.748 & 0.748 & 0.809 & 0.832 & 0.832 \\
\hline Model 9 & 1.771 & 1.796 & 1.780 & 2.259 & 2.284 & 2.271 \\
\hline Model 10 & 0.695 & 0.703 & 0.702 & 0.756 & 0.777 & 0.777 \\
\hline Model 11 & 1.840 & 1.831 & 1.828 & 2.359 & 2.349 & 2.345 \\
\hline Model 12 & 0.715 & 0.726 & 0.726 & 0.810 & 0.812 & 0.811 \\
\hline \multicolumn{7}{|c|}{ City-Block distance } \\
\hline Model & STAR & VAR & $\mathrm{AR}$ & & & \\
\hline Model 7 & 0.973 & 1.009 & 1.009 & & & \\
\hline Model 8 & 1.030 & 1.062 & 1.062 & & & \\
\hline Model 9 & 3.154 & 3.169 & 3.154 & & & \\
\hline Model 10 & 0.974 & 0.986 & 0.985 & & & \\
\hline Model 11 & 3.285 & 3.269 & 3.265 & & & \\
\hline Model 12 & 1.039 & 1.041 & 1.041 & & & \\
\hline \multicolumn{7}{|c|}{ STAR processes with $n=2$} \\
\hline & \multicolumn{6}{|c|}{ MAE } \\
\hline & \multicolumn{2}{|c|}{ STAR } & \multicolumn{2}{|c|}{ VAR } & \multicolumn{2}{|c|}{$\mathrm{AR}$} \\
\hline Model & $X_{\mathbf{s}_{L}}$ & $X_{\mathbf{s}_{U}}$ & $X_{\mathbf{s}_{L}}$ & $X_{\mathbf{s}_{U}}$ & $X_{\mathbf{s}_{L}}$ & $X_{\mathbf{s}_{U}}$ \\
\hline Model 13 & 1.569 & 1.467 & 1.569 & 1.467 & 1.569 & 1.467 \\
\hline Model 14 & 0.621 & 0.301 & 0.620 & 0.300 & 0.546 & 0.300 \\
\hline Model 15 & 0.631 & 0.365 & 0.633 & 0.363 & 0.628 & 0.361 \\
\hline Model 16 & 0.596 & 0.354 & 0.595 & 0.352 & 0.595 & 0.352 \\
\hline Model 17 & 0.650 & 0.330 & 0.657 & 0.330 & 0.651 & 0.328 \\
\hline Model 18 & 0.586 & 0.362 & 0.589 & 0.359 & 0.588 & 0.359 \\
\hline
\end{tabular}


Table 1 (cont.). Predictive Performance of Different Approaches

RMSE

\begin{tabular}{ccccccc}
\hline \multicolumn{4}{c}{ STAR } & \multicolumn{2}{c}{ VAR } & \multicolumn{2}{c}{$\mathrm{AR}$} \\
\hline Model & $X_{\mathbf{s}_{L}}$ & $X_{\mathbf{s}_{U}}$ & $X_{\mathbf{s}_{L}}$ & $X_{\mathbf{s}_{U}}$ & $X_{\mathbf{s}_{L}}$ & $X_{\mathbf{s}_{U}}$ \\
\hline Model 13 & 1.879 & 1.758 & 1.879 & 1.758 & 1.879 & 1.758 \\
Model 14 & 0.754 & 0.366 & 0.752 & 0.364 & 0.364 & 0.631 \\
Model 15 & 0.766 & 0.444 & 0.768 & 0.442 & 0.439 & 1.904 \\
Model 16 & 0.726 & 0.430 & 0.725 & 0.428 & 0.724 & 0.428 \\
Model 17 & 0.787 & 0.401 & 0.793 & 0.401 & 0.786 & 0.399 \\
Model 18 & 0.695 & 0.439 & 0.716 & 0.436 & 0.714 & 0.435 \\
\hline & Hausdorff distance & Euclidean distance \\
\hline Model & STAR & VAR & AR & STAR & VAR & AR \\
\hline Model 13 & 1.589 & 1.588 & 1.588 & 2.152 & 2.152 & 2.152 \\
Model 14 & 0.666 & 0.665 & 0.665 & 0.730 & 0.727 & 0.728 \\
Model 15 & 0.706 & 0.707 & 0.702 & 0.781 & 0.782 & 0.777 \\
Model 16 & 0.637 & 0.636 & 0.636 & 0.724 & 0.722 & 0.722 \\
Model 17 & 0.717 & 0.725 & 0.718 & 0.783 & 0.790 & 0.783 \\
Model 18 & 0.622 & 0.626 & 0.624 & 0.695 & 0.716 & 0.715 \\
\hline Model 15 & 0.996 & 0.995 & 0.989 & & & \\
\hline Model 16 & 0.950 & 0.947 & 0.947 & & & \\
\hline Model 17 & 0.982 & 0.987 & 0.979 & & & \\
\hline Model 18 & 0.944 & 0.948 & 0.947 & & & \\
\hline Model 13 & 3.036 & 3.036 & 3.036 & & & \\
Mock distance & & & & \\
\hline
\end{tabular}

